EMOTIONAL MEMORY IN PREGNANT WOMEN 


\title{
EMOTIONAL MEMORY IN PREGNANT WOMEN AT RISK FOR POSTPARTUM DEPRESSION
}

\author{
By MARISSA WILLIAMS, B.A.
}

A Thesis Submitted to the School of Graduate Studies in Partial Fulfillment of the Requirements for the Degree Master of Science 
McMaster University MASTER OF SCIENCE (2013) Hamilton, Ontario (Neuroscience)

TITLE: Emotional Memory in Pregnant Women at Risk for Postpartum Depression

AUTHOR: Marissa Williams, B.A. (McMaster University)

SUPERVISOR: Dr. Benicio N. Frey

NUMBER OF PAGES: viii, 98 


\begin{abstract}
Postpartum depression (PPD) is a serious disorder associated with debilitating effects on mothers and their infants. A previous history of depression appears to be the strongest risk factor for PPD. Previous studies showed that individuals with history of depression accurately recall more negative compared to positive content. The objective of this study was to compare emotional memory for negative and positive images between pregnant women with previous depressive episodes and pregnant women with no lifetime depression. This is the first study to investigate emotional memory in pregnant women with or without previous history of Major Depressive Disorder (MDD). A total of 77 participants between the ages of 18 - 44 (mean age: $27.3 \pm 6.2$ yo) completed the study (14 pregnant women with previous depressive episodes, 30 pregnant women with no lifetime depression, 13 non-pregnant women with previous depressive episodes, and 20 nonpregnant healthy). Participants took part in an emotional encoding task consisting of positive, negative, and neutral images from the International Affective Picture System (IAPS) where they were asked to rate these images based on perceived emotional intensity. Participants returned a week later for a surprise incidental recognition memory task. A multivariate general linear model revealed a significant main effect of group $(F(1,71)=8.04, p=.01)$. Women with history of MDD demonstrated poorer memory performance than women with no history for negative images, but the two groups did not differ on memory for positive images. This suggests that having a history of depression selectively impaired the memory recognition of negative images.
\end{abstract}




\section{Acknowledgments}

Thank you to all who were involved in this research including Drs. Benicio Frey, Sue Becker, Margaret McKinnon, Luciano Minuzzi, and Lauren Cudney and Marg Coote. I would like to express my very great appreciation to the midwives at Community Midwives of Hamilton for enabling me to visit the clinic and recruit their pregnant

clients. Finally, I would like to thank Lorenda Williams, John Williams, and Eric Johnson for their continued support. 


\section{Table of Contents}

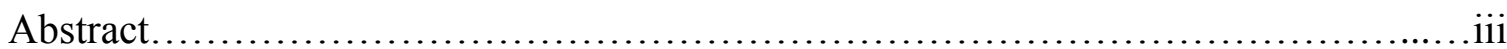

Acknowledgments.........................................................

Table Captions................................................................

Figure Captions.............................................................vii

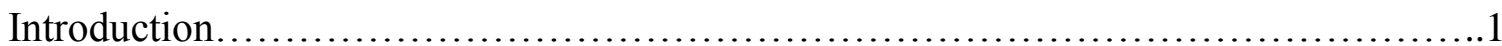

Objectives and Hypotheses................................................ 13

Method.........................................................................

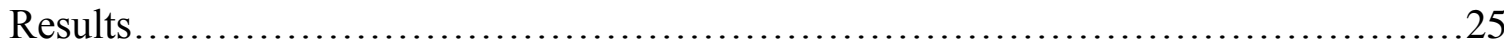

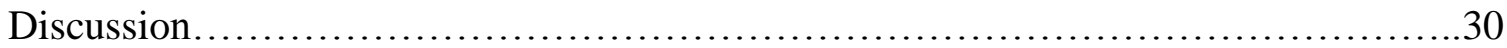

References................................................................... 43

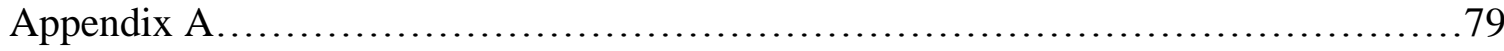

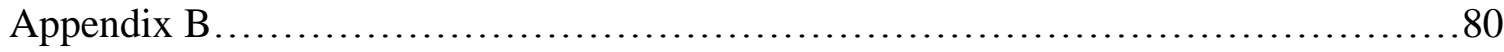

Appendix C.............................................................. 81

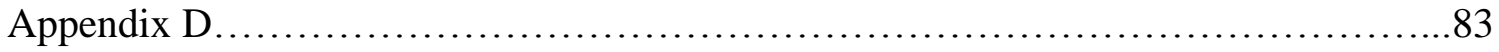


Appendix E............................................................ 85

Appendix F........................................................... 87 
M.Sc. Thesis - M. Williams; McMaster University - Neuroscience

\section{Table Captions}

Table 1. Demographic and Clinical Characteristics of Participant Groups..............60

Table 2. Depressive Symptoms as Assessed by EPDS during Pregnancy $(N=44)$ and at

Postpartum $(N=30)$ in Pregnant Groups.........................................62 
M.Sc. Thesis - M. Williams; McMaster University - Neuroscience

\section{Figure Captions}

Figure 1. a) Mean intensity ratings for each emotional valence category within participant groups

Figure 1. b) Mean intensity ratings for each valence category between pregnant $(N=44)$ and non-pregnant $(N=33)$ groups

Figure 1. c) Mean intensity ratings for each valence category between previous history of $M D D(N=27)$ and no previous history of $M D D(N=50)$ groups

Figure 2. a) Mean normalized memory sensitivity indexes (d') for emotional valence categories across participant groups........

Figure 2. b) Mean normalized memory sensitivity scores for emotional valence categories across pregnant $(N=44)$ and non-pregnant $(N=33)$ participant groups

Figure 2. c) Mean normalized memory sensitivity scores for emotional valence categories across participants with $(N=27)$ and without $(N=50)$ history of MDD

Figure 3. a) Total salivary-free cortisol secretion $\left(A U C_{G}\right)$ at baseline (time -1) in pregnant women with history of $M D D(n=14)$

Figure 3. b) Area under the cortisol curve $\left(A U C_{G}\right)$ for pregnant women with history of $M D D$ 
M.Sc. Thesis - M. Williams; McMaster University - Neuroscience

Figure 3. c) Area under the cortisol curve $\left(A U C_{G}\right)$ for pregnant women with history of MDD at age of gestation. .71

Figure 3. d) Total salivary-free cortisol secretion $\left(A U C_{G}\right)$ at baseline (time -1) in nonpregnant women with history of $M D D(n=12)$...

Figure 3. e) Area under the cortisol curve $\left(A U C_{G}\right)$ for non-pregnant women with $M D D$ history.....

Figure 3. f) Total salivary-free cortisol secretion $\left(A U C_{G}\right)$ at baseline (time -1) in pregnant healthy women $(n=28)$.

Figure 3. g) Area under the cortisol curve $\left(A U C_{G}\right)$ for pregnant healthy women.... .75

Figure 3. h) Area under the cortisol curve $\left(A U C_{G}\right)$ for pregnant healthy women at age of gestation.

Figure 3. i) Total salivary-free cortisol secretion $\left(A U C_{G}\right)$ at baseline (time -1) in nonpregnant healthy women $(n=20)$.

Figure 3. j) Area under the cortisol curve $\left(A U C_{G}\right)$ for non-pregnant healthy women .....78 


\section{Introduction}

Postpartum depression (PPD) is a common and preventable disorder associated with a debilitating effect on mothers and their infants (Wisner, Chambers, \& Sit, 2006). PPD must be distinguished from the "baby blues", which is mild and transient and affects $50-80 \%$ of new mothers (Henshaw, 2003). PPD is more serious and persistent whereas baby blues is commonly experienced in new mothers and lasts usually a week after giving birth (O’Hara, 2009). PPD is classified not as a discrete disorder, but as a sub-type of a Major Depressive Disorder (MDD) in The Diagnostic and Statistical Manual of Mental Disorders ( $5^{\text {th }}$ ed.; DSM-5; American Psychiatric Association, 2013) with an onset of a Major Depressive episode (MDE) within 4 weeks postpartum (O’Hara, 2009). Its core criterion symptoms include a depressed mood most of the day, nearly every day for duration of at least 2 weeks or longer and/or a loss of interest or pleasure in activities usually enjoyed. In addition, five or more diagnostic symptoms may include (but are not limited to) a sleep deficit beyond what is typical for care of the baby, lack of or excessive concern for the baby, obsessive thoughts or impulses with regard to the child, ambivalent feelings or lack of feeling for the child, fear of failure or feelings of inadequacy as a mother, drastic change in appetite, anxiety or irritability, weight loss or weight gain, psychomotor agitation (i.e., uncomfortable inability to remain still) or retardation (i.e., slowing of cognitive and/or physical activity), fatigue or loss of energy, feelings of worthlessness or guilt, poor concentration and suicidal ideations or acts (American Psychiatric Association, 2013). Women have approximately a 50\% higher risk of developing depression postnatally than at any other time in their lives (Vesga-Lopez, 
Blanco, Keyes, Olfson, Grant, \& Hasin, 2008). PPD is associated with higher rates of hospitalization, and increased risk of maternal suicide and infanticide (Comtois, Schiff, \& Grossman, 2008; Diego, Field, Hernandez-Reif, Cullen, Schanberg, \& Kuhn, 2004; Lindahl, Pearson, \& Colpe, 2005). Studies have shown that children of depressed mothers have reduced cognitive, emotional, and social skills and display an insecure-avoidant attachment to their mother (Brand \& Brennan, 2009). This is likely due to the mother's lack of response to their infant which does not allow them to bond or form secure attachments. Pearson, Lightman, and Evans (2009) found that women depressed during pregnancy showed less response to images depicting distressed infants compared to nondepressed women. Longitudinal studies have shown that children with mothers who suffered from PPD had elevated cortisol and norepinephrine levels, and developmental deficits in language, intelligence, and memory compared to children of non-depressed mothers (Cogill, Caplan, Alexandra, Robson, \& Kumar, 1986; Essex, Klein, Cho, \& Kalin, 2002; Grace, Evindar, Stewart, 2003). Additionally, offspring of mothers with depression are at increased risk of developing depressive and other psychiatric disorders. Research looking at maternal depression and risk for child psychopathology has found an association between mothers with recurrent depression and co-occurring psychiatric disorders such as anxiety or alcohol abuse and heightened risk for future psychiatric illness in offspring (Sellers, Collishaw, Rice, Thapar, Potter, \& Mars et al., 2012). A number of psychosocial and environmental risk factors for PPD have been identified including lack of social support, stressful life events, low self-esteem, lack of support from partner, prenatal anxiety, difficult temperament of child, and low socioeconomic 
status (Beck, 2001). In addition, previous history of depression, especially depression experienced prenatally, appears to be the strongest risk factor for PPD (Beck, 2001). This is evidenced by two large prospective studies (Heron, O’Connor, Evans, Golding, \& Glover, 2004; Milgrom, et al., 2008) that followed women during pregnancy and in the postpartum period and found that one of the strongest predictors for PPD is having a previous history of depression. PPD is estimated to affect between 7-15\% of women in the general population (O'Hara \& Swain, 1996). This rate is $25 \%$ among women with past postpartum episodes, putting pregnant women with a previous history of MDD at significantly greater risk for PPD (Wisner, Parry, \& Piontek, 2002).

\section{Memory}

In humans, it is a widely accepted view that emotional information is more accurately remembered than non-emotional information. This is because enhanced memories for positive and negative emotional experiences serve an important function for human behaviour (Christianson, 1987). Evolutionarily, stimuli that evoke positive or negative emotional and physiological arousal are more crucial and relevant to human survival than neutral stimuli (Pratto \& John, 1991). Thus, it is advantageous to store emotional information as accurately as possible as this leads to a greater probability of survival. Emotion substantially influences and enhances the vividness and longevity of memories, especially for episodic memory (Pratto \& John, 1991). Emotional memory can be defined as "memory for emotional stimuli, events or situations" (van Stegeren, 2008). 
The advent of neuroimaging techniques such as Positron Emission Tomography (PET) and Functional Magnetic Resonance Imaging (fMRI) has enabled researchers to examine the neural mechanisms involved in emotional information processing (Phan, Wager, Taylor, \& Liberzon, 2002). Many neuroimaging studies of humans have shown that there is increased amygdala activation following the presentation of emotional images, which is associated with better memory for these images. The amygdala works in concert with many interconnected brain regions such as the hippocampus, dorsal and ventral prefrontal cortex (PFC), thalamus, and hypothalamus to facilitate emotional learning and memory (Canli, Zhao, Desmond, Glover, \& Gabrieli, 1999; LaBar \& Cabeza, 2006). Due to their close proximity and direct neural projections to one another (i.e., the amygdala is located just anterior to the hippocampus, which is located in the medial temporal lobe), the amygdala enhances or alters the quality of hippocampal-dependent memory formation, organization, and storage for emotionally salient events through modulation of the hippocampus (Steinvorth, Levine, \& Corkin, 2005). Evidence to support this comes from neuroimaging studies of humans (Dolcos, LaBar, \& Cabeza, 2004; Kensinger \& Corkin, 2004) that have reported a significant correlation between the responsivity in the amygdala and hippocampus during encoding of emotional content. Evidence from animal studies has shown that glucocorticoids are important for memory enhancement of emotional information to occur. The amygdala mediates glucocorticoid effects on memory consolidation of recent emotional experiences via interactions with the hippocampus, which is enriched with glucocorticoid receptors (Roozendaal, 2000). The facilitating effect of stress hormones on memory performance will be discussed next. 


\section{Memory and stress hormones}

Hans Selye, known as the "father of stress" originally coined the term "stress" as related to pressures from the environment on biology (Selye, 1936). The major stress systems in the body are the sympathetic nervous system and the hypothalamic-pituitaryadrenal (HPA) axis, with the HPA axis being at the center of the stress response. The two major chemical messengers mediating the stress response are epinephrine and glucocorticoids (cortisol in humans, corticosterone in other animals). Corticotropinreleasing factor/hormone $(\mathrm{CRF} / \mathrm{CRH})$ is secreted by the paraventricular nucleus $(\mathrm{PVN})$ of the hypothalamus in response to stress, which in turn stimulates the secretion of adrenocorticotrophic hormone $(\mathrm{ACTH})$ and release of glucocorticoids. Cortisol, the primary glucocorticoid in humans, is a reliable biological marker for HPA activity whose levels increase in response to a stressor. Strong evidence has shown that the release of noradrenaline, a neurotransmitter and stress hormone, and glucocorticoids have differential effects on the consolidation and retrieval phases of memory. Specifically, they have impairing effects on subsequent memory retrieval when released during learning (Buchanan \& Tranel, 2008; de Quervain, Roozendaal, Nitsch, McGaugh, \& Hock, 2000), and enhancing effects on memory when released post-learning (i.e., during consolidation) (Andreano \& Cahill, 2006; Cahill, Gorski, \& Le, 2003). The consolidation of memory can be modulated by the interaction of noradrenaline and cortisol following an emotional task (Przybyslawski, Roullet, \& Sara, 1999; Nader, Schafe, \& Le Doux, 2000). Strong evidence from animal studies has shown that glucocorticoid enhancement of memory requires endogenous noradrenergic activation in the basolateral nucleus of the amygdala 
(BLA) in response to an arousing, emotional event. These studies have identified an important role of the noradrenergic system and the integrity of the BLA in emotional memory. Studies using rats show that noradrenergic agonists lead to enhanced memory performance on inhibitory avoidance tasks when injected in the BLA. In contrast, these memory effects can be reversed using a noradrenergic antagonist such as a betablocker, which leads to decreased memory performance (Roozendaal, 2000). To see if these effects were also shown in humans, Cahill, Prins, Weber, and McGaugh (1994) investigated the effect of propranolol hydrochloride, a $\beta$-adrenergic receptor antagonist, on long term memory for an emotional and neutral story. The researchers found that propranolol selectively impaired memory for the emotional story, but not the neutral story. These results show that memory for emotionally arousing information involves adrenergic mechanisms within an intact amygdala, as blocking the noradrenaline receptors with a beta-blocker such as propranolol significantly reduces memory for this information. However, a study by Segal and Cahill (2009) showed that higher levels of endogenous adrenergic and glucocorticoid release during encoding of an emotional memory task results in enhanced memory at retrieval for emotional stimuli compared to non-emotional stimuli. These researchers found a positive correlation between salivary alpha-amylase (sAA), an established biological marker of noradrenergic reactivity, and memory for emotionally arousing pictures in healthy individuals. These results strongly indicate that an increase in adrenergic activation is selective for the long-term memory of emotional content (Segal \& Cahill, 2009), and thus illustrates the discrepancy in the literature on the impairing and enhancing effects of cortisol on memory retrieval. A study 
by Buchanan and Lovallo (2001) looked at the effect of elevated cortisol levels on longterm memory performance by administering either cortisol or placebo to participants, and then having them view both emotionally arousing and emotionally neutral images. One week later participants' memory for the images was tested. The researchers hypothesized that the participants randomly assigned to receive exogenous administration of cortisol before stimulus presentation would display better memory one week later for the emotionally arousing images, but not for the emotionally neutral images. Results supported this hypothesis; those who received cortisol remembered more emotionally arousing images than those who received a placebo. These findings document the crucial involvement of cortisol in the formation of long term memory for emotional images through its enhancing influence on acquisition and consolidation. Taken together, it is clear that the amygdala and its noradrenergic receptors in the BLA play a pivotal role in

glucocorticoid mediated emotional learning and memory (Cahill, Babinski, Markowitsch, \& McGaugh, 1995; Phelps, 2004).

\section{Memory in depression}

It is well documented in the literature that individuals with clinical and subclinical depression recall less overall, and have enhanced memory for negative compared to positive content (Watkins, Matthews, Williamson, \& Fuller, 1992; Bradley, Mogg, \& Williams, 1995). This memory bias towards negative emotions is known as the "moodcongruent memory bias" and is theorized by some to be a maladaptive cognitive schema most commonly seen in individuals with depression (Kovacs \& Beck, 1978; Ridout, 
Astell, Reid, Glen, \& O'Carroll, 2003; Watkins et. al., 1992). Although the exact pathophysiology remains unknown, many brain regions and their interconnections involved in processing and regulating emotional behaviour, learning and memory, mood, motivation and reward have been implicated in the neural circuitry of depression (Beck, 2008; Murrough, Iacoviello, Neumeister, Charney, \& Iosifescu, 2011). These include the hippocampus, anterior cingulate cortex, and frontal cortex (e.g., prefrontal cortex) as well as several subcortical structures such as the nucleus accumbens, amygdala, hypothalamus, insula and other related limbic structures. Extensive research shows that the amygdala is hyperactive in depression (Hamilton \& Gotlib, 2008), and it has been posited by several investigators that there is over-activation of the amygdala-hippocampus system in response to negative affective stimuli in individuals with depression $(\mathrm{Fu}, \mathrm{Williams,}$ Cleare, Brammer, Walsh, Kim, Andrew, Pich, Williams, Reed, Mitterschiffthaler, Suckling \& Bullmore, 2004; Hamilton \& Gotlib, 2008; Sheline, Barch, Donnelly, Ollinger, Snyder, \& Mintun, 2001; Siegle, Steinhauer, Thase, Stenger, \& Carter, 2002; Surguladze, Brammer, Keedwell, Giampietro, Young, Travis, Williams, \& Phillips, 2005). This may be related to abnormalities within certain brain structures and/or disruption in the frontal-subcortical (i.e., "top down") pathways such that the prefrontal cortical areas are unable to "calm" or control the amygdala during emotional processing (Clark, Chamberlain, \& Sahakian, 2009). Hamilton and Gotlib (2008) were among the first researchers to examine the neural correlates of enhanced emotional recall for negative stimuli in individuals with depression. In an fMRI scanner, they presented control and depressed participants with positive, neutral, and negative images as part of a 
picture encoding task. Pictures for the task were selected from the International Affective Picture System (IAPS) (Lang, Bradley, \& Cuthbert, 1997) based on normative valence ("the positive or negative nature of a stimulus) and arousal ("the stimulating or calming nature of a stimulus”) (Mickley Steinmetz, Addis, Kensinger, 2010, p. 318) ratings. Participants were instructed on screen to rate the IAPS images based on perceived emotional intensity. One week after the scan, participants returned to the lab for an incidental recognition memory task, which assesses implicit memory for images, where original pictures shown during the encoding task were shown plus foil images not previously shown. Participants were asked to indicate whether they had seen each of the images by pressing one of three buttons: the number 1 key if they remembered not having seen the picture before, the number 2 key if the picture looked familiar or the number 3 key if they remembered they had seen the picture the previous week. Hamilton and Gotlib (2008) hypothesized that those individuals with depression would exhibit increased memory sensitivity for the negative images due to an over-recruitment of brain areas that are involved in memory for affective information during encoding. Indeed, the researchers found that individuals with MDD displayed a greater memory bias than nondepressed individuals for negative pictures, but not for positive or neutral pictures. In contrast, individuals with no lifetime history of depression recalled positive pictures more accurately than the negative pictures. The fMRI scan showed that this enhanced memory for negative stimuli at retrieval was associated with increased amygdala activation at encoding, and that the magnitude of the amygdalar response was dependent on the severity of depressive symptoms reported by depressed participants (Hamilton \& Gotlib, 
2008). These results suggested that there is greater activation of the amygdala for stimuli that are emotionally arousing, but not for neutral stimuli, and that this memory effect is selective for individuals with depression for the encoding of negative information (Hamilton \& Gotlib, 2008).

\section{Memory during pregnancy}

Pregnant women often report memory difficulties during pregnancy (Sharp, Brindle, Brown, \& Turner, 1993) including forgetfulness, absentmindedness, difficulties in concentrating, and decreased attention. Although there is some objective research to support this claim, many studies that have investigated whether these subjective pregnancy related memory impairments reflect objective deficits have yielded inconsistent results. The domains of memory that have been objectively found to be negatively affected by pregnancy are explicit episodic memory, implicit memory, semantic memory, working memory, and prospective memory, but these deficits are nowhere near as robust as the subjective reports from pregnant women (Cuttler, Graf, Pawluski, \& Galea, 2011). It is possible that the societal stereotype that pregnancy is frequently associated with memory deficits (often referred to as "baby brain") may contribute to pregnant women attributing more of their memory difficulties to pregnancy (Crawley, 2002; Crawley, Grant, \& Hinshaw, 2008). Overall, studies suggest that there are less severe objective memory deficits during pregnancy than what is subjectively reported by pregnant women. The focus in the literature has shifted to investigating 
potential explanations for these pregnancy related memory deficits. Pregnancy is marked by major physiological, psychological, and hormonal changes which are widely known to negatively affect memory (Cuttler et al., 2011). Previous studies have looked at whether hormones, specifically cortisol, may be responsible for the cognitive deficits accompanying pregnancy. Cortisol is known to significantly increase throughout pregnancy (Allolio, Hoffman, Linton, Winkelmann, Kusche, \& Schulte, 1990) and to adversely affect memory (Seeman, McEwan, Singer, Albert, \& Rowe, 1997; Heffelfinger $\&$ Newcomer, 2001). Despite this, previous studies that examined the possibility that cortisol may account for the memory difficulties reported in pregnancy have not found any evidence to support this (Buckwalter et al., 1999). Another possibility explored in the literature is that low mood and anxiety may negatively affect memory since these have also been reported during pregnancy (Jarrahi-Zadeh, Kane, Van De Castle, Lachenbrunch, \& Ewing, 1969; Keenan et al., 1998; Buckwalter et al., 2001). The literature appears to be inconsistent again, with some studies concluding that the mood changes that occur during pregnancy are related to subjective (Gross \& Pattison, 1994; Morris, Toms, Easthope, \& Biddulph, 1998) and objective reports (Jarrahi-Zadeh et al., 1969) of cognitive deficits, and others finding that mood changes do not relate to either subjective or objective reports (Poser, Kassirer, \& Peyser, 1986; Keenan et al., 1998; Buckwalter et al., 1999; Vanston \& Watson, 2005). This contradictory evidence leaves the question open as to whether or not mood fluctuations are an adequate explanation for the cognitive deficits pregnant women experience. Finally, pregnant women in the $3^{\text {rd }}$ trimester of pregnancy often report physical discomfort (e.g., backache, fatigue) and reduced sleep quality 
(Janes, Casey, Huntsdale, \& Angus, 1999; Crawley, 2002), which may have negative consequences for memory. Research has found that sleep quality, a clinical construct made up of components including sleep latency, sleep duration, habitual sleep efficiency, and sleep disturbances (Buysse, Reynolds, Monk, Berman, \& Kupfer, 1989) is related to subjective (Janes et al., 1999), but not objective memory deficits (Janes et al., 1999). Not all changes observed during pregnancy are negative however. Contrary to this common perception past research has shown that cognitive function in the domain of recognition performance (Mickes, Wixted, Shapiro, \& Scarff, 2009) and word list learning (Silber, Almkvist, Larsson, \& Uvnas, 1990) is actually enhanced during pregnancy. One study investigated emotionally influenced recognition for faces during pregnancy (Pearson et. al., 2009). These researchers used a facial recognition task to study pregnant women's ability to encode emotional faces in early gestation (7-14 weeks) and again at late gestation (33-39 weeks). Their findings show that pregnant women in late gestation had an enhanced ability to recognize facial expressions displaying fear, disgust, and anger compared to in early gestation, but no change in the ability to recognize sad or happy facial expressions, which the authors suggest reflects a hypervigilant emotion processing system during pregnancy for emotional stimuli signalling threat or harm. This makes sense from an evolutionary perspective, in that pregnant women would be selectively attentive to negative emotional stimuli if this enhanced recognition serves a protective and survival function for their offspring. There is the possibility that late pregnancy enhances memory for threatening stimuli because late in pregnancy there is an elevation of cortisol therefore leading to more efficient recognition of these stimuli. An enhanced 
memory for emotional negative content in pregnant women with a previous history of depression may be associated with an increased risk for PPD. Emotional memory has not been systematically investigated in pregnant women as evidenced by the lack of significant data reported in this understudied area (Pearson et al., 2009). To the best of our knowledge, our study is the first to investigate emotional memory in pregnant women with or without previous history of MDD.

The objective of the present study was to compare emotional memory in pregnant women considered to be high risk for PPD (those with previous depressive episodes but currently euthymic) with that of pregnant women considered at low risk (those with no lifetime depression). To control for potential pregnancy effects on emotional memory, non-pregnant women with and without previous depressive episodes were also recruited. The research hypotheses of this study are threefold. The primary hypothesis is that pregnant women with history of MDD will exhibit enhanced memory sensitivity for negative images as compared to pregnant women with no history of MDD. Similarly, the secondary hypothesis is that non-pregnant women with history of MDD will exhibit enhanced memory sensitivity for negative images as compared to non-pregnant women with no lifetime MDD. The third hypothesis is that memory sensitivity for negative stimuli would significantly correlate with increased levels of salivary cortisol (sCORT) and salivary alpha-amylase (sAA) in pregnant and in non-pregnant women with history of MDD. 


\section{Method}

\section{Participants}

The Research Ethics board of St. Joseph's Healthcare Hamilton approved the study. To date, 86 female participants have been recruited by a variety of methods including community advertisements, Experimetrix, a web-based experiment sign-up website, the Women's Health Concerns Clinic (WHCC) at St. Joseph's Healthcare Hamilton and a community midwives clinic in Hamilton, Ontario, Canada. A total of 470 individuals were screened for the study. The three most common reasons for study refusal were a lack of interest in the research, the minimum 2.5 hour study time commitment, and/or a busy schedule. Volunteers eligible and willing to take part in the study were selected after an initial screening interview. They were informed that they would be participating in a study examining the potential link between PPD and emotion.

Participants were informed that the experimental testing would occur on two separate occasions and that both would involve similar tasks. Pregnant participants were studied in the second trimester (between 12 to 22 weeks of gestational age) because this period is associated with the least reported physical discomfort and/or pain complaints and less sleep disturbance, which may influence emotional memory, compared to that seen in the third trimester of pregnancy (Orff \& Parry, 2013). Women with a previous history of depression all met DSM-IV criteria for MDD and had been euthymic for at least 3 months prior to study participation. All participants read and gave informed written consent. Participants were excluded if they met current criteria for any Axis I disorder. Additional 
exclusion criteria included: lifetime history of Schizophrenia, Schizoaffective Disorder, Delusional Disorder, Bipolar Disorder, Eating Disorder, or alcohol substance dependence; presence of any neurological disease; history of head trauma with loss of consciousness for more than five minutes; mental retardation; unstable medical condition; history of any anxiety disorder in the past six months; history of alcohol or substance abuse in the last 6 months. Participants were also deemed not eligible if they had Edinburgh Postnatal Depression Scale (EPDS) scores greater than 12. Exclusion criteria were selected to minimize confounds to emotional memory. All participants had an estimated Intelligence Quotient (IQ) of 70 or greater. Of the individuals initially enrolled in the study, 4 were deemed not eligible after administering The Structured Clinical Interview for DSM-IV Axis I Disorders (SCID-I/P) (First, Spitzer, Gibbon, \& Williams, 2002) and meeting lifetime criteria for an eating disorder and/or current criteria for Generalized Anxiety Disorder (GAD). Data were collected between July 2011 and May 2013. Data was excluded from 5 participants who failed to return for the second session. A total of 77 participants between the ages of $18-44$ (mean age: $27.3 \pm 6.2$ yo) successfully completed the study. This included 14 pregnant women with a history of major depressive episodes, 13 non-pregnant women with no history of major depressive episodes, 30 pregnant healthy women with no lifetime depression, and 20 non-pregnant healthy women. All non-pregnant participants verbally reported having regular menstrual cycles and were asked when the first day of their last menstrual period was. This was to ensure that testing was done during the follicular phase of the menstrual cycle to control for the potential influence of ovarian sex hormones on emotional memory (Ertman, Andreano, \& Cahill, 
2011). All participants were medication free, except for 4 participants ( 2 pregnant with previous history of MDD, 1 non-pregnant with previous history of MDD, and 1 nonpregnant healthy) whom reported taking Synthroid, and 4 participants (2 pregnant with previous history of MDD and 2 non-pregnant with previous history of MDD) who reported taking antidepressant medication (Cipralex or Effexor) at the time of the study. All groups were matched by age, total number of years of education, IQ score, gestational age (GA) and number of previous MDEs (when applicable).

\section{Materials}

The Structured Clinical Interview for DSM-IV Axis I Disorders (SCID-I/P) (First et al., 2002) was used to evaluate participants' current and/or past history of Axis I disorders. Depressive symptoms in the past week were assessed using the Edinburgh Postnatal Depression Scale (EPDS) (Cox et al., 1987) and the Montgomery-Asberg Depression Rating Scale (MADRS) (Montgomery \& Asberg, 1979). Both clinical scales are shown in Appendices A and B, respectively. The State-Trait Anxiety Index (STAI) (Spielberger \& Lushene, 1970) was administered to measure current state (i.e., anxiety about an event) and trait (i.e., anxiety as a personal characteristic) anxiety levels (see Appendix C). The Postpartum Depression Predictors Inventory-Revised (PDPI-R) (Beck, 2002) was used to determine risk factors present during pregnancy (Appendix D). Sleep quality was assessed using the Pittsburgh Sleep Quality Index (PSQI) (Buyesse et al., 1989) (Appendix E). All of these questionnaires are widely used and have shown high internal reliability and validity. To estimate baseline intellectual capacity (IQ score), two 
subtests (vocabulary and matrix reasoning performance) of the Wechsler Abbreviated Scale of Intelligence (WASI) (Weschler, 1999) were administered to all participants. Premorbid intellectual functioning was assessed using the Wechsler Test of Adult Reading (WTAR) (Wechsler, 2001). A standardized emotional reactivity task (ERT) included images selected from the IAPS (Lang, Bradley \& Cuthbert, 1997) and were divided into positive, neutral, and negative categories based on IAPS valence and arousal norms (as defined earlier). Positive pictures had a mean normed valence of 7.53 with a range between 6.56-8.59, and a mean normed arousal of 5.28; neutral pictures had a mean normed valence of 5.17 with a range between 4.53-5.76, and a mean normed arousal equal to 3.42; negative pictures had a mean normed valence of 2.66 with a range between 1.29-4.23, and a mean normed arousal of 5.65. Since the current study sample includes members of potentially vulnerable populations (i.e., pregnant women and women with depression), images were chosen with caution so as to not cause undue distress or mental anguish. A standardized emotional memory task (EMT) is the most commonly used memory test to investigate memory for emotional stimuli. Stimulus presentation, timing, and recording of behavioural data during the ERT and EMT were generated by an ASUS K53SV laptop with $37.8 \times 25.3 \times 2.83 \mathrm{~cm}$ dimensions and 15.6 inch display. ERT and EMT were coded using E-prime v1.2 software and administered according to van Stegeren et al. (2005). ERT and EMT tasks were used to measure emotional intensity ratings and emotional memory performance, respectively, for each valence category in participant groups. Please refer to Appendix F for emotional task instructions. Sorbettes (Salimetrics LLC, State College, PA, USA) were used to collect the sCORT and sAA 
saliva samples. The fraction of free cortisol was measured by an expanded range high sensitivity salivary cortisol enzyme immunoassay kit and alpha-amylase concentrations were determined using a kinetic enzyme assay kit, both with proven reliability and validity (Salimetrics LLC, State College, PA, USA).

\section{Procedures and Measures}

During the first session, consent was obtained and participants were assessed using the SCID-I/P (First et al., 2002) by a psychiatrist or trained graduate student. Participants completed self-reported (EPDS and STAI) and professionally administered (MADRS, PDPI-R, PSQI) questionnaires. The WTAR and WASI were then administered by a trained professional to all participants. Cortisol and alpha-amylase in saliva were collected at four different times during the first visit: 10 minutes before the emotional reactivity task (time -1), immediately before the task (time 0 ), immediately after the task (time 1), and 10 minutes after the task (time 2). Participants were asked to refrain from consuming caffeine and alcohol, or participating in cardiovascular exercise 24 hours prior to both study visits to control for diurnal hormonal variations (Segal \& Cahill, 2009; Walsh, Blannin, Clark, Cook, Robson \& Gleeson, 1999). Testing was conducted between the hours of 10:00 and 19:00 hours. At each time point, a saliva sample was collected by inserting a Sorbette under the participant's tongue for a minimum of 90 seconds. After the first saliva sample was given, participants were shown instructions on screen and completed a practice run before beginning the ERT task. Immediately after the second 
saliva sample, participants viewed a total of 144 images in random order, consisting of 48 neutral pictures, 48 negative pictures, and 48 positive pictures. A fixation cross was shown on screen for $500 \mathrm{~ms}$ before each image, which participants were told to focus on. Each picture was presented for 3000ms. Participants were instructed to view each picture for the entire time that it was on the screen. After the presentation of each picture, participants were asked on screen to indicate the emotional intensity of the picture on a 7point likert scale where 1 represented "not emotional at all" and 7 represented "extremely emotional". Immediately after completing the ERT, the third saliva sample was collected, then after 10 minutes the final saliva sample was collected. All saliva samples were stored at $-80^{\circ} \mathrm{C}$ until assayed. Participants were not aware at any time during the first visit that their memory would be assessed in the subsequent week. Rather, they were told they would return the following week to perform "a similar task". Participants returned one week later for the second session and once again completed the EPDS, MADRS, STAI, and PSQI questionnaires. Scores on these four questionnaires from the first session and second session were averaged so that any changes in mood, anxiety, and sleep quality could be accounted for. Participants also took part in an incidental recognition memory task (Segal \& Cahill, 2009) of which they had no previous knowledge. Participants viewed a total of 216 images, which included the 144 original images shown during the first session, and 72 foil pictures ( 24 pictures for each valence category) that were not previously shown. Foil pictures were matched for average valence and arousal to the original 144 images. Participants viewed each image and were then asked on screen to indicate whether they had seen the picture previously by pressing one of three buttons. 
Participants were instructed to press the number 1 key if they had not seen the picture before, the number 2 key if the picture looks familiar, but they are not sure, or the number 3 key if they are sure they had seen the picture before. Participants were debriefed on the purpose of the task and asked if they had any further questions following completion of the EMT.

\section{Pilot Follow-up Study}

This pilot follow-up study was added to the project after $19 \%$ of participants had completed their participation in the study. Pregnant and non-pregnant participants were individually contacted once over the telephone between 8-12 weeks postpartum or 28-32 weeks after the second study session, respectively. Follow-up times were determined based on estimated due dates and date of study completion to ensure pregnant and nonpregnant individuals were contacted at comparable times after the EMT sessions. The postpartum telephone follow-up assessment lasted approximately 5-10 minutes and consisted of re-administering the EPDS and the past MDE module of the SCID-I/P (First et al., 2002) to assess whether or not depressive symptoms and/or episodes had developed in any of the participants since study completion. 


\section{Data Analysis}

Statistics were performed using the Statistical Package for Social Sciences version 21.0 for Windows (IBM Corp., Armonk, NY, USA). A total of 77 participants were included in these analyses after excluding 5 participants who failed to return for the second study session. Differences between groups on age, education, IQ score, weeks of GA and number of previous MDEs (when applicable) were analyzed using one-way analyses of variance (ANOVA). For each participant, mean emotional intensity ratings and memory sensitivity indexes (d') were calculated for each of the three valence categories. Individual trials from the ERT were categorized according to the average intensity rating for each valence category, ranging from 1 ("not emotional at all”) to 7 ("extremely emotional"). A multivariate general linear model included intensity ratings with participant group (pregnancy status or MDD history) as the between-subjects factor and intensity ratings as a function of valence (negative, neutral, positive) as the withinsubjects factor. Here, 'pregnancy status' refers to whether participants are pregnant or non-pregnant and 'MDD history' refers to whether participants have a history of MDD or no history of MDD. A main effect of group (pregnancy status or MDD history) and the interaction between pregnancy status and MDD history were analyzed. Age and total number of years of education were included as covariates in the model. For the EMT, "Hits" were counted as the number of correctly identified original pictures rated as 2 (“familiar") or 3 ("with certainty") for each valence category. "False alarms" were counted as incorrectly recognizing a foil picture as having been shown during the first session (assigning a rating of "2" or " 3 "). Hit and False Alarm rates were calculated for 
each individual participant by dividing the number of hits and false alarms, respectively, by the total number of " 2 " and " 3 " responses for each category of emotional valence (Hamilton \& Gotlib, 2008). These rates were then used to calculate the sensitivity indexes (d'). Since it has been reported that depressed individuals remember less information overall in comparison to non-depressed individuals (Burt, Zembar, \& Niederehe, 1995), valence specific sensitivity indexes (positive and negative) were calculated by dividing the d' for positive and d' for negative information by the d' for neutral information, respectively, to control for variance explained by this reliable memory finding. A multivariate general linear model included these resultant memory sensitivity indexes with participant group (pregnancy status or MDD history) as the between-subjects factor and valence (positive, negative) as the within-subjects factor. A main effect of group (pregnancy status or MDD history) and the group interaction (pregnancy status by MDD history) was examined. Age, total number of years of education, and area under the cortisol curve $\left(\mathrm{AUC}_{\mathrm{G}}\right)$, which is the plot of cortisol versus time, were included as covariates in the model. To see whether intensity ratings for the emotional categories of images (negative, positive) during the first session correlated with memory performance for negative images on the second session one week later, multiple linear regression analyses were performed separately for those participants with a previous history of MDD and for participants without history of MDD. Memory performance for negative images was the dependent variable and intensity ratings for negative and positive images were included as predictors in the model. A Levene's test of equality of error variance was performed to assess whether participant groups had equal variances. A Shapiro-Wilk test 
was used to calculate the probability that the data are normally distributed for each participant group. The null hypothesis of this test is that the participant samples were drawn from a normally distributed population. The alternative hypothesis is that the participant samples were not drawn from a normally distributed population. Significance of post hoc comparisons between groups was calculated with the Bonferroni correction. All tests were based on a $95 \%$ confidence interval corresponding to an $\alpha$ of 0.05 to indicate statistical significance. Area under the curve (AUC) was used to incorporate the four time points of hormonal collection and detect individual and group changes in salivary cortisol and salivary alpha-amylase over time. Two formulas that are used for computation of the AUC are termed 'area under the curve with respect to ground' $\left(\mathrm{AUC}_{\mathrm{G}}\right)$ and 'area under the curve with respect to the increase' $\left(\mathrm{AUC}_{\mathrm{I}}\right)$ (Pruessner, Kirschbaum, Meinlschmid, \& Hellhammer, 2003), and are derived using trapezoidal integration (Reinhardt \& Soeder, 2001). In endocrinological research, the $\mathrm{AUC}_{\mathrm{G}}$ is useful when looking at total hormonal release whereas the $\mathrm{AUC}_{\mathrm{I}}$ is more useful in research designs using acute stress tasks such as the Trier Social Stress Test (TSST) (Kirschbaum, Pirke, \& Hellhammer, 1993) and the Cold Pressor Task (CPT) (van Stegeren, Wolf, \& Kindt, 2008) where levels of sAA are expected to increase and change over time. Since the current study did not administer a stressor, only AUCG $_{\text {was employed. Total release }}$ $\left(\mathrm{AUC}_{\mathrm{G}}\right)$ was calculated from the four salivary cortisol and four salivary alpha-amylase measures using 10 minute (between time -1 and time 0 , between time +1 and time +2 ) and 30 minute intervals (between time 0 and time +1 ) between measurements. The interval between measurements at time 0 and time +1 was 30 minutes as this was the 
duration of the ERT. The formula used to calculate $\mathrm{AUC}_{\mathrm{G}}$ for each individual participant is as follows:

$\mathrm{AUC}_{\mathrm{G}}=\left(\mathrm{m}_{2}+\mathrm{m}_{1}\right) * \mathrm{t}_{1} / 2+\left(\mathrm{m}_{3}+\mathrm{m}_{2}\right) * \mathrm{t}_{2} / 2+\left(\mathrm{m}_{4}+\mathrm{m}_{3}\right) * \mathrm{t}_{3} / 2$

with $t_{1}$ to $t_{3}$ denoting the time intervals between the single measurements and $m_{1}$ to $m_{4}$ representing the single measurements. The relation between total salivary-free cortisol and alpha-amylase secretion $\left(\mathrm{AUC}_{\mathrm{G}}\right)$ and subsequent memory performance was estimated using multiple linear regression. For each participant group, memory performance for negative images was the dependent variable and education, age, IQ score, and all original $\mathrm{AUC}_{\mathrm{G}}$ values for cortisol and alpha-amylase were included as predictors in the model. Additionally, Pearson product-moment correlation coefficients were computed to assess the strength of the linear association between time of day and the first cortisol sample (time-1) and between time of day and area under the cortisol curve $\left(\mathrm{AUC}_{\mathrm{G}}\right)$ for each participant group. A two-way ANOVA with repeated measures was used to analyze within and between pregnant group differences in EPDS scores during the second trimester of pregnancy and in the postpartum period. Participant group (pregnant with previous history of MDD or pregnant without previous history of MDD) was the between-subjects factor and EPDS as a function of time (12-22 weeks of GA or 8-12 weeks postpartum) was the within-subjects factor. A main effect of time, group, and their interaction (i.e., time by group) was examined. Age, total number of years of education, IQ score, weeks of GA and total number of MDEs were included as covariates in the model. We also examined the number of participants who met diagnostic criteria for a MDE as indicated by the SCID-I/P (First et al., 2002). 


\section{Results}

\section{Demographic and clinical characteristics}

Demographic and clinical characteristics are shown in Table 1. The 4 groups did not differ with respect to IQ score $(F(3,74)=1.0, p=.40)$, weeks of GA $(F(1,41)=1.01$, $p=.32$ ) or number of previous MDEs $(F(1,25)=.003, p=.96)$ (when applicable). However, there were significant differences between groups on age $(F(3,76)=6.97, p<.001)$ and total number of years of education $(F(3,76)=5.68, p=.002)$ as shown by one-way ANOVAs. A post hoc Bonferroni test revealed that the non-pregnant healthy group was younger and had fewer number of years of education completed than the pregnant with previous history of MDD ( $p=.001)$ and the pregnant healthy $(p=.002)$ groups. Thus, in order to control for these group differences, age and education were included as covariates in all statistical analyses. There were significant differences between groups on STAI state $(F(3,76)=6.71, p<.0001)$ and STAI trait $(F(3,76)=5.80, p=.001)$ scores, but not on PSQI $(F(3,76)=.97, p=.41)$ or $\operatorname{MADRS}(F(3,76)=2.02, p=.12)$ scores. A post hoc Bonferroni test revealed that the non-pregnant with previous history of MDD group had higher STAI state scores than non-pregnant healthy $(p=.045)$ and pregnant healthy $(\mathrm{p}<.0001)$ groups. Similarly, the non-pregnant with previous history of MDD group had higher STAI trait scores than the pregnant healthy group $(p=.001)$. 


\section{Emotional Intensity Ratings}

The mean intensity ratings for emotional valence categories across all participant groups are displayed in Figures 1a-c. A multivariate linear regression analysis revealed that the general linear model was marginally significant for intensity ratings for positive images $(F(5,71)=2.31, p=.05)$. The main effect of group (pregnancy status or MDD history) was not significant; pregnant and non-pregnant groups did not differ on intensity ratings for negative $(F(1,71)=.19, p=.66)$, neutral $(F(1,71)=1.16, p=.29)$ or positive $(F(1,71)=.18, p=.67)$ images. Similarly, groups with or without a previous history of MDD did not differ on intensity ratings for negative $(F(1,71)=.17, p=.68)$, neutral $(F(1,71)=.16, p=.70)$ or positive $(F(1,71)=.01, p=.91)$ images. There was a significant interaction between the effects of pregnancy status and MDD history on emotional intensity ratings for negative $(F(1,71)=4.52, p=.04)$ and positive $(F(1,71)=8.68, p=.004)$, but not neutral $(F(1,71)=.83, p=.37)$ images. In pregnant women, having a previous history of MDD was associated with higher intensity ratings for negative and positive images. In non-pregnant women, history of MDD was associated with lower intensity ratings for these same emotional categories of images. 


\section{Memory Sensitivity (d')}

The mean normalized memory sensitivity indexes (d') for emotional valence categories across all groups are shown in Figures 2a-c. A multivariate linear regression revealed that the general linear model was marginally significant for memory sensitivity for negative images $(F(5,71)=2.11, p=.08)$. The main effect of group (pregnancy status) was not significant; pregnant and non-pregnant groups did not differ in memory performance for negative $(F(1,71)=1.02, p=.32)$ or positive $(F(1,71)=.002, p=.96)$ images. However, there was a main effect of group (MDD history); groups with or without a previous history of MDD differed in memory performance for negative $(F(1,71)=8.04, p=.01)$, but not positive $(F(1,71)=2.40, p=.13)$ images. Women with history of MDD had poorer memory performance than women with no previous history of MDD for negative, but not positive images. The interaction between pregnancy status and MDD history on memory performance for negative $(F(1,71)=.33, p=.57)$ and positive $(F(1,71)=1.04, p=.31)$ images was not significant. The relation between intensity ratings for negative and positive images on the first session and memory performance for negative images on the second session was estimated using multiple linear regression. Memory performance for negative images was not predicted by intensity ratings for negative images, $\mathrm{b}=.008, t(.11), p=.91$ or positive images, $\mathrm{b}=.022, t(.22), p=.83$. 


\section{Salivary cortisol and alpha-amylase}

Of the 77 participants, data were missing due to insufficient saliva collection in two $(2.5 \%)$ participants. Pearson correlation coefficients revealed that there was a negative correlation between salivary cortisol at baseline (10 minutes before the ERT) and time of day in the pregnant $\left(\mathrm{r}_{\mathrm{P}}=-.40, p=.04\right)$ and non-pregnant $\left(\mathrm{r}_{\mathrm{P}}=-.57, p=.008\right)$ healthy groups, but not in pregnant $\left(\mathrm{r}_{\mathrm{P}}=-.17, p=.56\right)$ and non-pregnant $\left(\mathrm{r}_{\mathrm{P}}=.03, p=.93\right)$ women with previous history of MDD. Similarly, total salivary-free cortisol secretion $\left(\mathrm{AUC}_{\mathrm{G}}\right)$ was negatively correlated with time of day in pregnant $\left(\mathrm{r}_{\mathrm{P}}=-.48, p=.012\right)$ and non-pregnant $\left(\mathrm{r}_{\mathrm{P}}=-.54, p=.02\right)$ healthy groups, but not in pregnant $\left(\mathrm{r}_{\mathrm{P}}=-.29, p=.31\right)$ and non-pregnant $\left(\mathrm{r}_{\mathrm{P}}=.16, p=.67\right)$ with previous history of MDD groups. Interestingly, total salivary-free cortisol secretion ( $\mathrm{AUC}_{\mathrm{G}}$ ) and weeks of GA were strongly correlated in pregnant healthy women $\left(\mathrm{r}_{\mathrm{P}}=.52, p=.004\right)$, but not in pregnant women with previous history of MDD ( $\left.\mathrm{r}_{\mathrm{P}}=-.22, p=.46\right)$. A line graph for each group summarizes these results in Figures 3a-j. The relation between memory performance for negative images and salivary hormones (cortisol and alpha-amylase $\mathrm{AUCG}_{\mathrm{G}}$ ) were estimated using multiple linear regression. Memory performance for negative images were not predicted by area under the cortisol curve $\left(\mathrm{AUC}_{\mathrm{G}}\right), \mathrm{b}=.05, t(.42), p=.67$ or area under the alpha-amylase curve $\left(\mathrm{AUC}_{\mathrm{G}}\right), \mathrm{b}=-.03, t(-.22), p=.82$. However, in this model MDD history significantly predicted memory performance for negative images, $\mathrm{b}=-.31, t(-2.57), p=.01$; MDD history explained $13 \%$ of variance in memory performance for the negative stimuli, $R^{2}=$ $.13, F(6,67)=1.61, p=.01$. These results from multiple linear regression confirms the previously reported multivariate general linear model finding that MDD history 
significantly predicted memory for negative images, and suggests that this effect is independent of salivary cortisol or alpha-amylase.

\section{Pilot Follow-up study}

Average EPDS scores for pregnant groups during the second trimester of pregnancy and in postpartum are presented in Table 2. To date, pilot follow-ups have been done on 42 women including 32 pregnant ( 9 with previous history of MDD and 23 with no history of MDD) and 10 non-pregnant participants (7 with previous history of MDD and 3 with no history of MDD). Three women (7\%) were unable to be contacted due to reasons that included moving out of the country $(\mathrm{N}=2)$, or not providing consent to be contacted at follow-up (N=1). Among the successful 42 follow-ups, 6 (14.3\%) women did meet diagnostic criteria for a MDE, while 36 (85.7\%) women did not. Among these 6 women who have met criteria for a MDE in the follow-up, $5(83.3 \%)$ had a previous history of MDD (1 pregnant and 4 non-pregnant). A two-way ANOVA with repeated measures (group over time) revealed that the main effect of EPDS as a function of time was significant for pregnancy and in the postpartum period $(F(1,28)=6.16, p=.02)$. Both pregnant groups had increased EPDS scores in postpartum compared to pregnancy. There was also a main effect of group on $\operatorname{EPDS}$ scores $(F(1,28)=8.77, p=.01)$. Univariate analyses revealed that the pregnant with previous history of MDD group had higher EPDS scores than the pregnant without previous history of MDD during pregnancy (12- 
22 weeks $)(F(1,42)=6.42, p=.02)$ and in postpartum $(8-12$ weeks $)(F(1,30)=5.24$, $p=.03)$. The EPDS by group interaction was not significant $(F(1,28)=.20, p=.66)$.

\section{Discussion}

To the best of our knowledge, this is the first study to systematically investigate emotional memory in pregnant women with or without a previous history of MDD. The objective of the present study was to compare emotional memory between pregnant women considered to be high risk for PPD (those with previous depressive episodes but currently euthymic) and pregnant women considered at low risk (those with no previous depressive episodes). We tested the hypothesis whether pregnant women with previous history of MDD had greater memory sensitivity for negative images compared to pregnant women without previous history of MDD. Similarly, we investigated whether non-pregnant women with history of MDD displayed enhanced memory for negative images compared to non-pregnant women without history of MDD. Our hypotheses were based on previous studies showing better recollection for negative content in individuals with depression. We also examined whether there was a relation between memory enhancement for negative images and increased levels of sCORT and sAA $\left(\mathrm{AUC}_{\mathrm{G}}\right)$ in the pregnant and non-pregnant women with history of MDD. This hypothesis is based on previous research that has shown that higher levels of adrenergic and glucocorticoid hormones during an emotional encoding task results in enhanced memory for emotional stimuli compared to non-emotional stimuli (Segal \& Cahill, 2009). 
We found that pregnant and non-pregnant groups did not differ on emotional intensity ratings for the three categories of images. This finding suggests that pregnancy did not affect the encoding or processing of these images in pregnant compared to nonpregnant women. Since female sex hormones (i.e., estrogen, progesterone) have been found to increase the sensitivity of emotion processing systems in women (Pearson et al., 2009) and pregnancy is associated with large increases in these hormones throughout gestation, it would be possible that these hormonal changes could enhance the processing of emotional stimuli in pregnant women, especially for negative stimuli (Pearson et al., 2009). Pearson et al. (2009) looked at changes in emotion processing from early (12 weeks of GA) to late pregnancy (37 weeks of GA) in healthy women and found that late gestation was associated with an enhanced ability to encode emotional expressions suggesting threat or harm (negative emotions in general) compared with early gestation. Since the current study included pregnant women with an average gestation of 17.9 weeks (mid pregnancy) this may partly explain why the pregnant women did not differ significantly from non-pregnant women on intensity ratings for the negative images especially.

Individuals with a previous history of MDD and individuals without history of MDD did not differ on intensity ratings for the three valence categories of images. We would expect to find a difference in intensity ratings for the neutral images between these two groups based on the literature that shows that depressed individuals are more likely to interpret neutral stimuli as negative and healthy individuals to interpret neutral stimuli as positive. Gollan, Pane, McCloskey, and Coccaro (2008) examined differences in affective 
information processing between healthy and currently depressed individuals using a facial recognition task and found that depression was related to interpreting neutral facial expressions as negative (i.e., sad) whereas healthy participants were more likely to interpret neutral facial expressions as positive (i.e., happy). Based on Gollan et al.'s (2008) findings one would expect these differences in the perception of neutral images to affect the emotional intensity ratings for these images. One could also expect these two groups to differ on intensity ratings for the negative images, based on cognitive biases observed in MDD (i.e., "distorted information processing or attentional allocation toward negative stimuli") (Murrough et al., 2011, p. 553). Beck's "cognitive triad", which is part of his cognitive theory of depression, posit that cognitive schemas direct attention and memory towards negative thoughts about the self, the world and the future in individuals suffering from depression (Beck, 2008; Beck, Rush, Shaw, \& Emery, 1978). These negative biases in cognitive processing gives rise to depressive symptoms and manifest in the form of thoughts and emotions. Since individuals with depression often show a persistent pattern of negative thinking, it would be expected that they would approach this task by selectively attending to the negative images because this negative pattern of thinking is hard wired.

We also found that a between-subjects factors (i.e., pregnancy status and MDD history) interaction on intensity ratings for the emotional images (negative, positive), but not the neutral images. In pregnant women, having a previous history of MDD was associated with higher intensity ratings for negative and positive images. This suggests that pregnancy during the $2^{\text {nd }}$ trimester enhances emotional reactivity to negative and 
positive images only in those women with a history of MDD. It is conceivable that in addition to having a previous history of depression, the state of pregnancy may add a significant evolutionary component. It is advantageous for pregnant women to be selectively attentive to negative stimuli that may signify threat or harm to the survival of her unborn child. It is beneficial for her to be hypervigilant to this type of stimuli in her environment in order to better protect her offspring. However, it is surprising that pregnant women with no history of depression would be less reactive to the positive images. It may be that more energy/attention/resources are needed to selectively attend to the negative as opposed to the positive images (which do not pose any real or potential threat) in favour of the negative images which pose as the greatest threat to survival for her and her unborn child. In non-pregnant women, history of MDD was associated with lower intensity ratings for the emotional image categories (negative, positive). This finding is not what you would expect based on the literature on the well-established cognitive bias of negative content in individuals with depression. One would expect women with a history of MDD to rate the negative images as more emotionally intense than the positive images.

We also found that pregnant and non-pregnant women did not differ in memory performance for negative and positive images. Most studies that have investigated memory performance during pregnancy have showed increased memory complaints in pregnant women in the $3^{\text {rd }}$ trimester of pregnancy with only two studies to date investigating whether cognitive functioning is altered in the $2^{\text {nd }}$ trimester of pregnancy (Keenan et al., 1998; Sharp et al., 1993). The current study measured emotional memory 
in the $2^{\text {nd }}$ trimester of pregnancy in pregnant women with or without a previous history of MDD, which has not been previously studied. Our finding suggests that pregnancy does not negatively affect emotional memory as commonly reported by pregnant women during the $3^{\text {rd }}$ trimester because recognition performance for the negative images was not worse, if anything, it was slightly enhanced in pregnant compared to non-pregnant women (although not significant). This finding is supported by past research that has shown that recognition performance is actually enhanced during pregnancy (Mickes et al., 2009; Pearson et al., 2009). However, this was found only in late third trimester whereas the pregnant women in the current study were in the second trimester. Our results may not have reached significance due to a number of reasons including low sample size, low statistical power, or the lack of inclusion of women during the third trimester.

In contrast to the findings in individuals with depression who display a memory bias for negative images and our primary hypothesis, women with a previous history of MDD displayed poorer memory performance for the negative images compared to women with no previous history of MDD, while the two groups did not differ in memory performance for the positive images. Therefore, having a history of MDD lead to decreased memory recall of negative images compared to not having a history. Although participants with a previous history of MDD had been euthymic for at least 3 months prior to study participation, we still expected to find that these individuals would remember more negative compared to positive images based on evidence that even when not currently depressed or in full remission from depression, memory for negative content still exists (Abela, Stolow, Zhang, \& McWhinnie, 2010). Abela et al. (2010) looked at the 
extent to which negative cognitive style was associated with having a previous history of depression in currently euthymic university students. These researchers used self-report questionnaires and a clinical interview to determine participants' current depressive symptoms and negative cognitive style, negative core beliefs, and current and past history of MDEs. Their hypothesis that negative cognitive style would be strongly associated with having a previous history of depression after controlling for current depressive symptoms was supported. This finding suggests that the pattern of negative thinking and emotions that is characteristic of clinical depression is still present in individuals who are not currently depressed. Possible reasons for this intriguing finding in the current study may be that the previously depressed individuals had learned effective cognitive coping strategies since they were all currently euthymic. For the pregnant previously depressed individuals, it may be that they had taken the necessary precautions to prevent a relapse in their depression during pregnancy. Since the majority of these women were recruited from a midwifery clinic, where low-risk pregnant women with a high socioeconomic status are typically seen, it may be that these women were highly educated on the effects of untreated depression on children's risk of impaired cognitive and emotional abilities and on perinatal mood disorders in general (Hubner-Liebermann, Hausner, \& Wittmann, 2012).

We found that intensity ratings for positive and negative images during the first study session did not subsequently predict memory performance for negative images during the second session one week later. This finding suggests that the mechanism by which individuals react to emotional stimuli is not related to emotional memory of these 
stimuli and that these may be different phenomena altogether. Based on the literature one would expect that if you are more reactive to an emotional stimulus you should have better memory for that stimulus, particularly when that stimulus is negatively-valenced.

Since it is well established in the literature that cortisol has a diurnal variation (Segal \& Cahill, 2009), changes in cortisol concentration relative to this circadian rhythm may relate to emotional enhancement (LaBar \& Cabeza, 2006) depending on the time of the day. We correlated time of day and cortisol levels measured at baseline, time of day and total salivary-free cortisol secretion $\left(\mathrm{AUC}_{\mathrm{G}}\right)$, and total salivary-free cortisol secretion $\left(\mathrm{AUC}_{\mathrm{G}}\right)$ and weeks of GA (where applicable). See Figures 3a-j for a line graph of these results. Interestingly, cortisol levels measured at baseline were negatively correlated with time of day in the pregnant and non-pregnant healthy groups, but not in pregnant and nonpregnant groups with MDD history. Similarly, total salivary-free cortisol secretion $\left(\mathrm{AUC}_{\mathrm{G}}\right)$ was negatively correlated with time of day in pregnant and non-pregnant healthy groups, but not in pregnant and non-pregnant groups with MDD history. Moreover, total salivary-free cortisol secretion $\left(\mathrm{AUC}_{\mathrm{G}}\right)$ and weeks of GA were strongly correlated in pregnant healthy women, but not in pregnant women with previous history of MDD. These findings have replicated previous literature showing that healthy individuals show a diurnal pattern of cortisol secretion such that there is a gradual decline of cortisol throughout the day (i.e., cortisol levels are highest in the early morning and lowest in the evening), whereas depressed individuals show a disruption in this diurnal rhythm in cortisol (Sachar, Hellman, Rofwarg, Halpern, Fukushima, \& Gallagher, 1973). With 
respect to the differences found in cortisol response between pregnant healthy and pregnant individuals with MDD history, no studies have previously studied or reported this. Most studies have examined the changes in salivary cortisol with advancing gestation in healthy pregnant women and have shown the expected diurnal decline in salivary cortisol across the day (Kivlighan, DiPietro, Costigan, \& Laudenslager, 2008), but not in pregnant women with a history of MDD. In the current study, it appears that having a history of MDD washes out the correlation between baseline cortisol and area under the cortisol curve $\left(\mathrm{AUC}_{\mathrm{G}}\right)$ with time of day and weeks of GA. The reported results therefore need to be replicated in future studies to allow firm conclusions to be drawn. In the linear regression model, area under the cortisol curve $\left(\mathrm{AUC}_{\mathrm{G}}\right)$ did not predict memory performance for negative images. Similarly, area under the alpha-amylase curve $\left(\mathrm{AUC}_{\mathrm{G}}\right)$ did not significantly predict memory performance. These findings are contrary to previous studies showing that elevated cortisol levels during memory encoding positively and selectively correlate with memory for emotional content (Buchanan \& Lovallo, 2001; Segal \& Cahill, 2009). However, MDD history explained $13 \%$ of variance in memory performance for the negative stimuli. This confirms the previously reported two-way ANOVA finding that MDD history significantly predicted poorer memory for negative images.

We have found from the pilot follow-up study data that $6(14.3 \%)$ women met criteria for a MDE, while $36(85.7 \%)$ women did not. These numbers are in accordance with the $7-15 \%$ prevalence of PPD in the general population. Both pregnant groups scored higher on the EPDS in postpartum than during pregnancy. This may be due to the 
new transition into motherhood (e.g., breastfeeding, early awakening of the infant therefore lack of sleep for the mother, lack of energy, low mood due to the natural drastic drop in progesterone and estrogen following birth, etc.). The pregnant with history of MDD group had significantly higher EPDS scores than the pregnant without history of MDD group during pregnancy (12-22 weeks) and in postpartum (8-12 weeks). Those with previous history of MDD were more likely to meet criteria for postpartum depression according to the SCID-I/P.

Several limitations must be considered when interpreting the results from our study. First, a large number of pregnant participants in the study (i.e., 34 women comprising $51 \%$ of the total sample size) were recruited from a community midwives clinic. Women who choose midwifery care may be qualitatively different than women who choose other forms of prenatal care such as obstetric care, leading to a more homogenous sample. One group was younger and, therefore, had less years of education compared to the other 3 groups. Because of this, age and total number of years of education were included as covariates in all statistical models to control for this. Second, non-pregnant participants verbally reported having regular menstrual cycles and being in the follicular phase of their cycle at time of study participation. The study did not measure hormone levels to confirm this, which may be a potential confounding variable as previous studies have shown that emotional information is better recalled during the highhormone luteal phase, with higher levels of progesterone subsequently predicting and mediating this enhanced emotional memory (Ertman, Andreano, \& Cahill, 2011). However, it is unlikely that participants would blatantly give an inaccurate report of when 
the first day of their last menstrual period was, for example, to choose a more desirable study date, as the experimenter was very flexible in terms of allowing participants to choose the dates that were the most convenient for them to take part in the study. Therefore, not confirming menstrual cycle phase by objectively measures is unlikely to have affected memory recall overall. Third, participants were asked to refrain from caffeine, alcohol, and cardiovascular exercise at least 24 hours prior to saliva collection in order to control for outside influences that may affect baseline salivary alpha-amylase levels. This information was ascertained by self-report and was not corroborated by objective measures. However, since it is recommended by health professionals to abstain from caffeine and alcohol intake during pregnancy, it is less likely that pregnant women in the current study consumed these and therefore unlikely that this would have affected baseline salivary alpha-amylase levels. Forth, it is possible that there may have been a priming effect for select pictures shown during the practice task since these pictures were shown in both the practice and ERT. This priming may result in facilitation in emotional memory performance due to previous exposure. Finally, Hamilton and Gotlib (2008) analyzed data using emotional contrast values to estimate effect size in their sample size of 26 individuals and found a 0.25 difference in positive and negative emotional sensitivity between healthy control and depressed groups. Due to the current study having only 77 participants completed at the time this manuscript was prepared, there were not enough participants in each group to achieve 80 percent power based on their results. Thirty participants per group were needed to reach this percentage of power to statistically compare differences between groups based on their results. Therefore, some 
statistical tests had low power due to the number of covariates that were controlled for in the model, which may have understated the true effect size for some of the results.

A future direction may be to use a longitudinal experimental design where you look at pre- and post-pregnancy measures of emotional memory. This will allow you to compare emotional memory before, during, and after pregnancy to see how the state of being pregnant affects emotional memory. Most studies that have investigated the impact of pregnancy on emotional memory have used a post hoc experimental design in that they compared memory in women who were already pregnant to when they were no longer pregnant or like the present study, assessed memory solely during pregnancy. This study design will surely be more causal in objectively determining whether/how pregnancy affects emotional memory. Future studies should also explore the possibility that having an emotional memory bias for emotionally negative images may be a cognitive marker of PPD in women with history of MDD. This cognitive marker may be present before the symptoms of depression develop. Therefore, emotional memory tasks may be useful as a preventative tool to screen and allow for early identification of PPD. Additionally, salivary cortisol and alpha-amylase levels may serve as a reliable biological marker to diagnose or predict PPD in longitudinal experimental designs with larger sample sizes than the current study. Identifying biomarkers for psychiatric disorder is an area of heavy research in psychiatry (Frank \& Hargreaves, 2003). In the context of PPD, elevated placental corticotropin-releasing hormone $(\mathrm{pCRH})$ as a potential biomarker of those at risk for perinatal or puerperal depression has been an area of recent interest. Three 
longitudinal studies have looked at the use of $\mathrm{pCRH}$ as a possible biomarker and found opposing results regarding the association between elevated pCRH levels and risk for prenatal and PPD (Meltzer-Brody, Stuebe, Dole, Savitz, Rubinow, \& Thorp, 2011; RichEdwards, Mohllajee, Kleinman, Hacker, Majzoub, Wright, \& Gillman, 2008; Yim, Glynn, Dunkel Schetter, Hobel, Chicz-DeMet, \& Sandman, 2009). Therefore, a reliable biological marker of risk for PPD in pregnant women has not yet been identified. Clearly, more work needs to be done in this understudied area.

Another future direction is to use neuroimaging techniques and emotionally valenced stimuli to help characterize the neuroanatomical abnormalities associated with dysregulated emotion in postpartum since the etiology of PPD remains unclear. Currently, PPD is classified as a specifier of a MDD in the DSM-5 (American Psychiatric Association, 2013). However, this continues to be questioned. There is evidence to suggest that there is differential amygdala activation in postpartum compared to nonpostpartum (unipolar) depression. Silverman, Loudon, Liu, Mauro, Leiter, \& Goldstein (2011) used fMRI to assess the neural processing of negative emotion in women with no lifetime history of depression 6-8 weeks postpartum. These researchers found a negative correlation between the lack of postpartum depressive symptoms as measured by the EPDS and increased right amygdala activation. There was less responsivity of the right amygdala in response to threatening vs. neutral stimuli in the women who developed PPD. In contrast, there was enhanced responsivity in women with non-postpartum depression. The finding that women with PPD have a blunted affect to negative stimuli 
compared to new mothers without PPD who are instead hypersensitive to negative stimuli provides insight into the potentially different neurophysiologic mechanisms responsible for depression in the postpartum period. This should be further explored in future studies in this area.

In conclusion, the objective of this study was to compare emotional memory in pregnant women considered to be high risk for PPD (those with previous depressive episodes) with pregnant women considered at low risk (those with no lifetime depression). Our results showed that pregnant women with a history of MDD did not exhibit enhanced memory for negative images compared to pregnant women without history. Rather, pregnant women with history of MDD displayed decreased memory for negative images. It appears that having a history of MDD predicted poorer memory performance for negative images. Area under the cortisol and alpha-amylase curve $\left(\mathrm{AUC}_{\mathrm{G}}\right)$ was not associated with memory performance for negative images in pregnant women with history of MDD. These findings are contrary to previous studies in healthy controls showing that elevated cortisol levels during memory encoding positively and selectively correlate with memory for emotional content. Future directions include using pre- and post-pregnancy measures of emotional memory, finding a reliable biological marker of risk for PPD, and using neuroimaging techniques and emotionally valenced stimuli to help characterize the neuroanatomical abnormalities associated with dysregulated emotion in postpartum to help clarify the etiology of PPD. 


\section{References}

Abela, J., Stolow, D., Zhang, M., \& McWhinnie, C. (2010). Negative cognitive style and past history of major depressive episodes in university students. Cognitive Therapy and Research, 1-9.

Allolio, B., Hoffman, J., Linton, E. A., Winkelmann, W., Kusche, M., \& Schulte, H. M. (1990). Diurnal salivary cortisol patterns during pregnancy and after delivery: relationship to plasma corticotrophin-releasing hormone. Clinical endocrinology (Oxford), 33(2), 279-289.

American Psychiatric Association. (2013). Diagnostic and statistical manual of mental disorders $\left(5^{\text {th }}\right.$ ed.). Washington, DC: Author.

Anderson, A.K., Yamaguchi, Y., Grabski, W., \& Lacka, D. (2006). Emotional memories are not all created equal: evidence for selective memory enhancement. Learning and Memory, 13, 711-718.

Andreano, J. M., \& Cahill, L. (2006). Glucocorticoid release and memory consolidation in men and women. Psychological science, 17(6), 466-470.

Beck, A. T. (2008). The evolution of the cognitive model of depression and its neurobiological correlates. The American Journal of Psychiatry, 165, 969-977. 
Beck, A.T., Rush, A.J., Shaw, B.F., \& Emery, G. (1979). Cognitive therapy of depression. New York: Guilford.

Beck, C. T. (2001). Predictors of postpartum depression: an update. Nursing research, 50, 275-285.

Beck, C.T. (2002). Revision of the postpartum depression Predictors Inventory. Journal of Obstetric, Gynecological, \& Neonatal Nursing, 31(4), 394-402

Borrell, J., de Kloet, E.R., Versteeg, D.H., \& Bohus, B. (1983). Inhibitory avoidance deficit following shortterm adrenalectomy in the rat: the role of adrenal catecholamines. Behavioural and neural biology, 39, 241-258

Bradley, B. P., Mogg, K., \& Williams, R. (1995). Implicit and explicit memory for emotion-congruent information in clinical depression and anxiety. Behaviour Research and Therapy, 33(7), 755-770.

Brand, S. R., \& Brennan, P. A., (2009). Impact of antenatal and postpartum maternal mental illness: how are the children? Clinical obstetrics and gynecology, 53, 441455. 
Buchanan, T. W., \& Lovallo, W. R. (2001). Enhance memory for emotional material following stress-level cortisol treatment in humans. Psychoneuroendocrinology, 26, 307-317.

Buchanan, T., W., \& Tranel, D. (2008). Stress and emotional memory retrieval: effects of sex and cortisol response. Neurobiology of learning and memory, 89, 134-141.

Buckwalter, J. G., Buckwalter, D. K., Bluestein, B. W., \& Stanczyk, F. Z. (2001). Pregnancy and post partum: changes in cognition and mood. Progress in brain research, 133, 303-319.

Buckwalter, J. G., Stanczyk, F. Z., McCleary C. A., Bluestein, B. W., Buckwalter, D. K., Rankin, K. P., Chang, L., \& Goodwin, T. M. (1999). Pregnancy, the postpartum, and steroid hormones: effects on cognition and mood. Psychoneuroendocrinolgy, 24(1), 69-84.

Burt, D. B., Zembar, M. J., Niederehe, G. (1995). Depression and memory impairment: A meta-analysis of the association, its pattern, and specificity. Psychological Bulletin, 117, 285-305. 
Buysse, D. J., Reynolds, C. F., Monk, T. H., Berman, S. R., \& Kupfer, D. J. (1989). The Pittsburgh Sleep Quality Index: a new instrument for psychiatric practice and research. Psychiatry Research, 28(2), 193-213.

Cahill, L., Gorski, L, \& Le, K. (2003). Enhanced human memory consolidation with postlearning stress: interaction with the degree of arousal at encoding. Learning and memory, 10(4), 270-274.

Cahill, L., Prins, B., Weber, M., \& McGaugh, J. L. (1994). Beta-adrenergic activation and memory for emotional events. Nature, 371(6499), 702-704.

Canli, T., Zhao, Z., Desmond, J., Glover, G., \& Gabrieli, J.D.E. (1999). FMRI identifies a network of structures correlated with retention of positive and negative emotional memory. Psychobiology, 27, 441-452.

Clark, L., Chamberlain, S. R., \& Sahakian, B. J. (2009). Neurocognitive mechanisms in depression: implications for treatment. Annual Review of Neuroscience, 32, 57-74.

Cogill, S. R., Caplan, H. L., Alexandra, H., Robson, K. M., \& Kumar, R. (1986). Impact of maternal postnatal depression on cognitive development of young children. British Medical Journal, 292(6529), 1165-1167.

Comtois, K. A., Schiff, M. A., \& Grossman, D. C. (2008). Psychiatric risk factors associated with postpartum suicide attempt in Washington State, 1992-2001. American Journal of Obstetrics and Gynecology, 199(2), 1-5. 
Cox, J. L., Holden, J. M., \& Sagovsky, R. (1987). Detection of postnatal depression. Development of the 10-item Edinburgh Postnatal Depression Scale. British Journal of Psychiatry, 150, 782-786.

Crawley, R.A. (2002). Self-perception of cognitive changes during pregnancy and the early postpartum: salience and attentional effects. Applied Cognitive Psychology, $16,617-633$.

Crawley, R.A., Grant, S., \& Hinshaw, K. (2008). Cognitive changes in pregnancy: mild decline or societal stereotype? Applied Cognitive Psychology, 22, 1142-1162.

Cuttler, C., Graf, P., Pawluski, J. L., \& Galea, L. A. (2011). Everyday life memory deficits in pregnant women. Canadian journal of experimental psychology, 65(1), 27-37.

de Quervain, D. J. F., Roozendaal, B., Nitsch, R. M., McGaugh, J. L., \& Hock, C. (2000). Acute cortisone administration impairs retrieval of long-term declarative memory in humans. Nature neuroscience, 3, 313-314.

Diego, M. A., Field, T., Hernandez-Reif, M., Cullen, C., Schanberg, S., \& Kuhn, C. (2004). Prepartum, postpartum, and chronic depression effects on newborns. Psychiatry, 67(1), 63-80.

Essex, M. J., Klein, M. H., Cho, E., \& Kalin, N. H. (2002). Maternal stress beginning in infancy may sensitize children to later stress exposure: Effects on cortisol and behavior. Biological Psychiatry, 52, 776-784. 
First, M. B., Spitzer, R. L., Gibbon, M., \& Williams, J. B. W. (2002). Structured Clinical Interview for DSM-IV-TR Axis-I Disorders, Patient Edition, (SCID-I/P) New York: Biometrics Research, New York State Psychiatric Institute

Frank, R., \& Hargreaves , R. (2003). Clinical biomarkers in drug discovery and development. Nature Reviews Drug Discover, 2, 566-580.

Fu, C. H., Williams, S. C., Cleare, A. J., Brammer, M. J., Walsh, N. D., Kim, J., Andrew, C. M., Pich, E. M., Williams, P. M., Reed, L. J., Mitterschiffthaler, M. T., Suckling, J., \& Bullmore, E. T. (2004). Attenuation of the neural response to sad faces in major depression by antidepressant treatment: a prospective, event-related functional magnetic resonance imaging study. Archives of general psychiatry, 61(9), 877-889.

Gollan, J. K., Pane, H. T., McCloskey, M. S., \& Coccaro, E. F. (2008). Identifying differences in biased affective information processing in major depression. Psychiatry Research, 159, 18-24.

Gozansky, W. S., Lynn, J. S., Laudenslager, M. L., \& Kohrt, W. M. (2005). Salivary cortisol determined by enzyme immunoassay is preferable to serum total cortisol for assessment of dynamic hypothalamic-pituitary--adrenal axis activity. Clinical Endocrinology, 63, 336-341. 
Grace, S. L., Evindar, A., Stewart, D. E. (2003). The effect of postpartum depression on child cognitive development and behaviour: a review and critical analysis of the literature. Archives of women's mental health, 6(4), 263-274.

Hamann, S. (2001). Cognitive and neural mechanisms of emotional memory. Trends in Cognitive Science, 5(9), 394-400.

Hamilton, J.P., \& Gotlib, I.H. (2008). Neural substrates of increased memory sensitivity for negative stimuli in major depression. Biological Psychiatry, 63, 1155-1162

Heffelfinger, A. K., \& Newcomer, J. W. (2001). Glucocoticoid effects on memory function over the human life span, Development and psychopathology, 13(3), 491513.

Henshaw, C. (2003). Mood disturbance in the early puerperium: a review. Archives of women's mental health, 6(2), 33-42.

Heron, J., O’Connor, T. G., Evans, J., Golding, J., \& Glover, V. (2004). The course of anxiety and depression through pregnancy and the postpartum in a community sample. Journal of Affective Disorders, 80(1), 65-73.

Hubner-Liebermann, B., Hausner, H., \& Wittmann, M. (2012). Recognizing and treating 
peripartum depression. Deutsches arzteblatt international journal, 109(24), 419424.

IBM Corp. Released 2011. IBM SPSS Statistics for Windows, Version 20.0. Armonk, NY: IBM Corp.

Janes, C., Casey, P., Huntsdale, C., \& Angus, G. (1999). Memory in pregnancy. I: Subjective experiences and objective assessment of implicit, explicit and working memory in primigravid and primiparous women. Journal of psychosomatic obstetrics and gynaecology, 20(2), 80-87.

Jarrahi-Zadeh, A., Kane, F. J. Jr., Van De Castle, R. L., Lachenbrunch, P. A., \& Ewing, J. A. (1969). Emotional and cognitive changes in pregnancy and early puerperium. The british journal of psychiatry: the journal of mental science, 115 (524), 797-805.

Keenan, P. A., Yaldoo, D. T., Stress, M. E., Fuerst, D. R., Ginsburg, K. A. (1998). Explicit memory in pregnant women. American journal of obstetrics and gynecology, 179(3 pt 1), 731-737.

Kirschbaum, C., Pirke, K. M., \& Hellhammer, D. H. (1993). The 'Trier Social Stress Test' - a tool for investigating psychobiological stress responses in a laboratory setting. Neuropsychobiology, 28(1-2), 76-81. 
Kivlighan, K. T., DiPietro, J. A., Costigan, K. A., \& Laudenslager, M. L. (2008). Diurnal rhythm of cortisol during late pregnancy: Associations with maternal psychological well-being and fetal growth. Psychoneuroendocrinology, 33, 1225-1235.

Kovacs, M., \& Beck, A. T. (1978). Maladaptive cognitive structures in depression. American Journal of Psychiatry, 135(5), 525-533.

LaBar, K. S., \& Cabeza, R. (2006). Cognitive neuroscience of emotional memory. Nature Reviews Neuroscience, 7, 54-64.

Lang, P.J., Bradley, M.M., \& Cuthbert, B.N. (1997). International Affective Picture System (IAPS): Technical manual and affective ratings. Gainseville, FL: University of Florida, Center for Research in Psychophysiology.

Lindahl, V., Pearson, J. L., \& Colpe, L. (2005). Prevalence of suicidality during pregnancy and the postpartum. Archives of women's mental health, 8(2), 77-87.

Lord, C., Hall, G., Soares, C., \& Steiner, M. (2010). Physiological stress response in postpartum women with obsessive-compulsive disorder: A pilot study. Psychoneuroendocrinology, 36(1), 133-138.

Meltzer-Brody, S., Stuebe, A., Dole, N., Savitz, D., Rubinow, D., \& Thorp, J. (2011). Elevated corticotropin releasing hormone $(\mathrm{CRH})$ during pregnancy and risk of 
postpartum depression (PPD). The Journal of clinical endocrinology and metabolism. 96(1), 40-47.

Micco, D.J., \& McEwen, B.S. (1980). Glucocorticoids, the hippocampus, and behavior: interactive relation between task activation and steroid hormone binding specificity. Journal of comparative and physiological psychology, 94, 624-633.

Mickes, L., Wixted, J. T., Shapiro, A., Scarff, J. M. (2009). The effects of pregnancy on memory: recall is worse but recognition is not. Journal of clinical and experimental neuropsychology, 31(6), 754-761.

Mickley Steinmetz, K. R., Addis, D. R., Kensinger, E. A. (2010). The effect of arousal on the emotional memory network depends on valence. Neuroimage, 53, 318-324.

Milgrom, J., et al. (2008). Antenatal risk factors for postnatal depression: a large prospective study. Journal of Affective Disorders. 108(1-2), 147-57.

Montgomery, S. A., \& Asberg, M. (1979). A new depression scale designed to be sensitive to change. British Journal of Psychiatry, 134, 382-329.

Morris, N., Toms, M., Easthope, Y., \& Biddulph, J. (1998). Mood and cognition in pregnant workers. Applied ergonomics, 29(5), 377-381. 
Murrough, J. W., Iacoviello, B., Neumeister, A., Charney, D. S., \& Iosifescu, D. V. (2011). Cognitive dysfunction in depression: neurocircuitry and new therapeutic strategies. Neurobiology of learning and memory, 96, 553-563.

Nader, K., Schafe, G. E., \& Le Doux, J. E. (2000). The labile nature of consolidation theory. Nature reviews neuroscience, 1(3), 216-219.

O’Hara, M. W. (2009). Postpartum depression: what we know. Journal of clinical psychology, 65(12), 1258-1269.

O'Hara, M. W., \& Swain, A. (1996). Rates and risk of postpartum depression - a metaanalysis. International Review of Psychiatry, 8, 37-54.

Orff, H. J., \& Parry, B. (2013). Pregnancy and Postpartum. Encyclopedia of Sleep, 292296.

Pearson, R. M., Lightman, S. L., \& Evans, J. (2009). Emotional sensitivity for motherhood: late pregnancy is associated with enhanced accuracy to encode emotional faces. Hormones and behavior, 56(5), 557-563. 
Phan, K. L., Wager, T., Taylor, S. F., \& Liberzon, I. (2002). Functional neuroanatomy of emotion: a meta-analysis of emotion activation studies in PET and fMRI. NeuroImage, 16, 331-348.

Phelps, E. (2004). Human emotion and memory: interactions of the amygdala and hippocampal complex. Current opinion in neurobiology, 14, 198-202.

Poser, C. M., Kassirer, M. R., \& Peyser, J. M. (1986). Benign encephalopathy of pregnancy. Preliminary clinical observations. Acta neurologica scandinavica, 73(1), 39-43.

Pratto, F., \& John, O. P. (1991). Automatic vigilance: The attention-grabbing power of negative social information. Journal of Personality and Social Psychology, 61, 380 $-391$.

Przybyslawsk, J., Roullet, P., \& Sara, S. (1999). Attenuation of emotional and nonemotional memories after their reactivation: role of $\beta$ adrenergic receptors. The journal of neuroscience, 19(15), 6623-6228.

PsychologicalCorporation. (1999). Wechsler Abbreviated Scale of Intelligence Manual. San Antonio, TX: Harcourt Assessment. 
PsychologicalCorporation. (2001). Wechsler Test of Abbreviated Reading Manual. San Antonio, TX Harcourt Assessment.

Reinhardt, F., \& Soeder, H. (2001). Dtv Atlas Mathematik. Deutscher Taschenbuch Verlag, Munchen

Rich-Edwards, J.W., Mohllajee, A.P., Kleinman, K., Hacker, M.R., Majzoub, J., Wright, R.J., \& Gillman, M.W. (2008). Elevated midpregnancy corticotropin-releasing hormone is associated with prenatal, but not postpartum, maternal depression. Journal of Clinical Endocrinology, \& Metabolism, 93, 1946-1951.

Ridout, N., Astell, A., Reid, I., Glen, T., \& O’Carroll, R. (2003). Memory bias for emotional facial expressions in major depression. Cognition \& Emotion, 17(1), 101-122.

Rohleder, N., Wolf, J. M., Maldonado, E. F., \& Kirschbaum, C. (2006). The psychosocial stress-induced increase in salivary alpha-amylase is independent of saliva flow rate. Psychophysiology, 43, 645-652.

Roozendaal, B. (2000). Glucocorticoids and the regulation of memory consolidation. Psychoneuroendocrinology, 25, 213-238. 
Sachar, E. J., Hellman, L., Roffwarg, H. P., Halpern, F. S., Fukushima, D. K., \& Gallagher, T. F. (1973). Disrupted 24-hour patterns of cortisol secretion in psychotic depression. Archives of General Psychiatry, 28, 19-24.

Seeman, T. E., McEwen, B. S., Singer, B. H. Albert, M. S., \& Rowe, J. W. (1997). Increase in urinary cortisol excretion and memory declines: MacArthur studies of successful aging. The journal of clinical endocrinology \& Metabolism, 82, 24582465

Segal, S. K., \& Cahill, L. (2009). Endogenous noradrenergic activation and memory for emotional material in men and women. Psychoneuroendocrinology, 34(9), 12631271.

Sellers, R., Collishaw, S., Rice, F., Thapar, A. K., Potter, R., Mars, B., Harold, G. T., Smith, D. J., Owen, M. J., Craddock, N., \& Thapar, A. (2012). Risk of psychopathology in adolescent offspring of mothers with psychopathology and recurrent depression. The British Journal of Psychiatry: The Journal of Mental Science, 202(2), 108-114.

Sharp, K., Brindle, P. M., Brown, M. W., \& Turner, G. M. (1993). Memory loss during pregnancy. British journal of obstetrics and gynaecology, 100(3), 209-215. 
Sheline, Y. I., Barch, D. M., Donnelly, J. M., Ollinger, J. M., Snyder, A. Z., \& Mintun, M. A. (2001). Increased amygdala response to masked emotional faces in depressed subjects resolves with antidepressant treatment: an fMRI study. Biological psychiatry, 50(9), 651-658.

Siegle, G. J., Steinhauer, S. R., Thase, M. E., Stenger, V. A., \& Carter, C. S. (2002). Can't shake that feeling: event-related fMRI assessment of sustained amygdala activity in response to emotional information in depressed individuals. Biological psychiatry, 51(9), 693-707.

Silber, M., Almkvist, O., Larsson, B., \&Uvnas, M. (1990). Temporary peripartal impairment in memory and attention and its possible relation to oxytocin concentration. Life Sci, 47, 57-65.

Spielberger, C. D. G., \& Lushene, R. E. (1970). Manual for the state-trait anxiety inventory. Palo

Alto, CA, USA: Consulting Psychologists Press

Surguladze, S., Brammer, M. J., Keedwell, P., Giampietro, V., Young, A. W., Travis, M. J., Williams, S. C., \& Phillips, M. L. (2005). A differential pattern of neural response toward sad versus happy facial expressions in major depressive disorder, Biological psychiatry, 57(3), 201-209. 
Van Stegeren, A. H. (2008). The role of the noradrenergic system in emotional memory. Acta Psychologica, 127(3), 532-541.

Van Stegeren, A.H., Goekoop, R., Everaerd, W., Scheltens, P., Barkhof, F., Kuijer, J.P.A, \& Rombouts, S.A.R. (2005). Noradrenaline mediates amygdala activation in men and women during encoding of emotional material. Neuroimage, 24, 898-909

Van Stegeren, A. H., Wolf, O. T., \& Kindt, M. (2008). Salivary alpha amylase and cortisol responses to different stress tasks: impact of sex. International journal of psychophysiology, 69(1), 33-40.

Vanston, C. M., \& Watson, N. V. (2005). Selective and persistent effect of foetal sex on cognition in pregnant women. Neuroreport, 16(7), 779-782.

Vesga-Lopez, O., Blanco, C., Keyes, K., Olfson, M., Grant, B.F., \& Hasin D.S. (2008). Psychiatric disorders in pregnant and postpartum women in the United States. Archives of General Psychiatry, 65(7), 805-815.

Walsh, N. P., Blannin, A. K., Clark, A.M., Cook, L., Robson, P.J., \& Gleeson, M. (1999). The effects of high-intensity intermittent exercise on saliva IgA, total protein and alpha-amylase. Journal of Sports Science and Medicine, 17, 129-134. 
Watkins, P. C., Matthews, A., Williamson, D. A., \& Fuller, R. D. (1992). Moodcongruent memory in depression: emotional priming or elaboration? Journal of Abnormal Psychology, 101(3), 581-600.

Wisner, K. L., Chambers, C., \& Sit, D. K. Y. (2006) Postpartum depression: a major public health problem. Journal of the American Medical Association, 296, 26122618.

Wisner, K. L., Parry, B. L., \& Piontek, C. M. (2002). Clinical practice. Postpartum depression. The New England Journal of Medicine, 347(3), 194-199.

Yim, I.S., Glynn, L.M., Dunkel Schetter, C., Hobel, C.J., Chicz-DeMet, A., \& Sandman, C.A.(2009). Risk of postpartum depressive symptoms with elevated corticotropinreleasing hormone in human pregnancy. Archives of General Psychiatry, 66(2), 162-169. 
Table 1

Demographic and Clinical Characteristics of Participant Groups

Group

\begin{tabular}{|c|c|c|c|c|c|}
\hline & $\begin{array}{l}\text { Pregnant \& } \\
\text { Previous } \\
\text { MDD }\end{array}$ & $\begin{array}{l}\text { Non-pregnant } \\
\text { Previous } \\
\text { MDD }\end{array}$ & $\begin{array}{l}\text { Pregnant } \\
\text { Healthy }\end{array}$ & $\begin{array}{l}\text { Non-pregnant } \\
\text { Healthy }\end{array}$ & $\mathrm{P}$ value \\
\hline Age, years & $31 \pm 4.4$ & $27 \pm 7.6$ & $29 \pm 4.2$ & $23 \pm 6.9$ & $.000 * * * a b$ \\
\hline $\begin{array}{l}\text { Education, } \\
\text { years }\end{array}$ & $17.3 \pm 3.5$ & $16.4 \pm 3.5$ & $16.9 \pm 3.1$ & $13.5 \pm 2.7$ & $\begin{array}{l}.006 * * \mathrm{a} \\
.003 * * \mathrm{~b}\end{array}$ \\
\hline $\begin{array}{l}\text { Gestational } \\
\text { age, weeks }\end{array}$ & $18.4 \pm 2.9$ & N/A & $17.6 \pm 2.5$ & N/A & .94 \\
\hline $\begin{array}{l}\text { Number of } \\
\text { MDEs }\end{array}$ & $2.9 \pm 5.0$ & $2.8 \pm 4.0$ & N/A & N/A & .83 \\
\hline MADRS & $3.4 \pm 2.7$ & $4.1 \pm 4.3$ & $2.45 \pm 1.9$ & $2.0 \pm 2.4$ & .12 \\
\hline $\begin{array}{l}\text { STAI, state } \\
\text { score }\end{array}$ & $27.8 \pm 5.5$ & $32.7 \pm 7.6$ & $25.1 \pm 3.2$ & $27.7 \pm 5.1$ & $\begin{array}{l}.000 * * * \mathrm{c} \\
.04 * \mathrm{~d}\end{array}$ \\
\hline $\begin{array}{l}\text { STAI, trait } \\
\text { score }\end{array}$ & $35.3 \pm 6.3$ & $38.3 \pm 9.2$ & $30.5 \pm 3.4$ & $32.8 \pm 6.2$ & $.001 * * \mathrm{c}$ \\
\hline $\begin{array}{l}\text { PSQI Global } \\
\text { score }\end{array}$ & $4.4 \pm 2.8$ & $5.7 \pm 3.7$ & $4.6 \pm 2.2$ & $4.2 \pm 1.9$ & .41 \\
\hline $\begin{array}{l}\text { IQ score, } \\
\text { WASI }\end{array}$ & $114.6 \pm 7.4$ & $109.9 \pm 16.1$ & $111.2 \pm 11.6$ & $107.7 \pm 13.7$ & .40 \\
\hline
\end{tabular}

Note. Mean \pm SD.

MADRS, Montgomery-Asberg Depression Rating Scale.

STAI, State-Trait Anxiety Inventory. 
PSQI, Pittsburgh Sleep Quality Index.

WASI, Wechsler Adult Intelligence Scale.

*. The mean difference is significant at the 0.05 level.

**. The mean difference is significant at the 0.01 level.

***. The mean difference is significant at the 0.001 level.

${ }^{a}$ Difference is significant between pregnant with previous history of MDD and non-pregnant healthy groups

${ }^{\mathrm{b}}$ Difference is significant between pregnant healthy and non-pregnant healthy groups

${ }^{c}$ Difference is significant between pregnant healthy and non-pregnant with previous history of MDD

${ }^{\mathrm{d}}$ Difference is significant between non-pregnant with previous history of MDD and non-pregnant healthy groups 
Table 2

Depressive Symptoms as Assessed by EPDS during Pregnancy $(N=44)$ and at Postpartum $(N=30)$ in Pregnant Groups

\section{Group}

Variable

$\begin{array}{lll}\text { Pregnant \& } & \text { Pregnant } & \text { P value } \\ \text { Previous } & \text { Healthy } & \\ \text { MDD } & \end{array}$

\begin{tabular}{llll}
\hline EPDS during & $5.0 \pm 2.67$ & $2.56 \pm 2.13$ & $.02 *$
\end{tabular}

pregnancy $^{\mathrm{a}}$

\begin{tabular}{llll}
\hline $\begin{array}{l}\text { EPDS at } \\
\text { postpartum }^{\text {b }}\end{array}$ & $5.67 \pm 1.94$ & $3.35 \pm 2.77$ & $.03 *$
\end{tabular}

Note. Mean $\pm \mathrm{SD}$.

EPDS, Edinburgh Postnatal Depression Scale.

*. The mean difference is significant at the 0.05 level.

a. 12-22 weeks of gestational age $(n=30$ healthy pregnant; $n=14$ pregnant with MDD history)

b. 8-12 weeks. ( $n=9$ pregnant with MDD history; $n=23$ healthy pregnant) 
Figure 1. a) Mean intensity ratings for each emotional valence category within participant groups

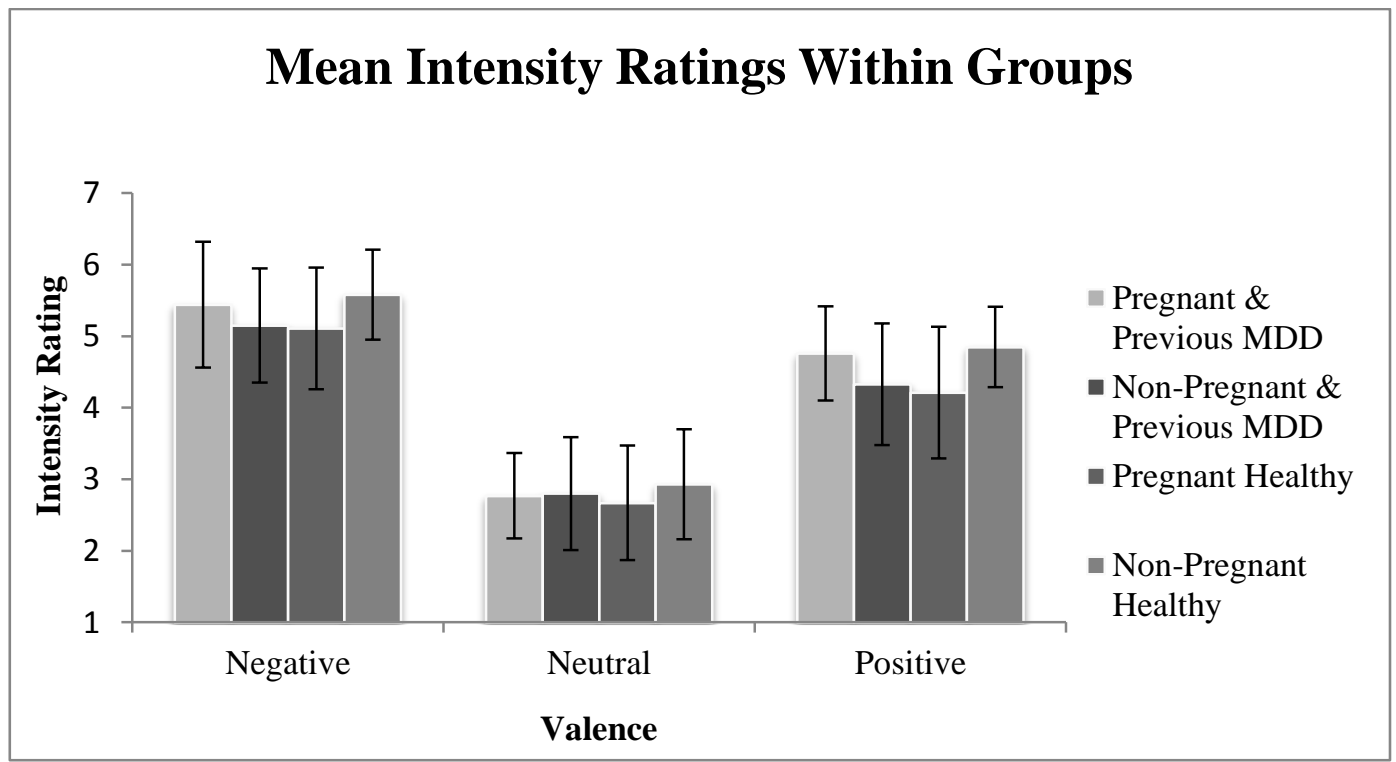


Figure 1. b) Mean intensity ratings for each valence category between pregnant $(\mathrm{N}=44)$ and non-pregnant $(\mathrm{N}=33)$ groups

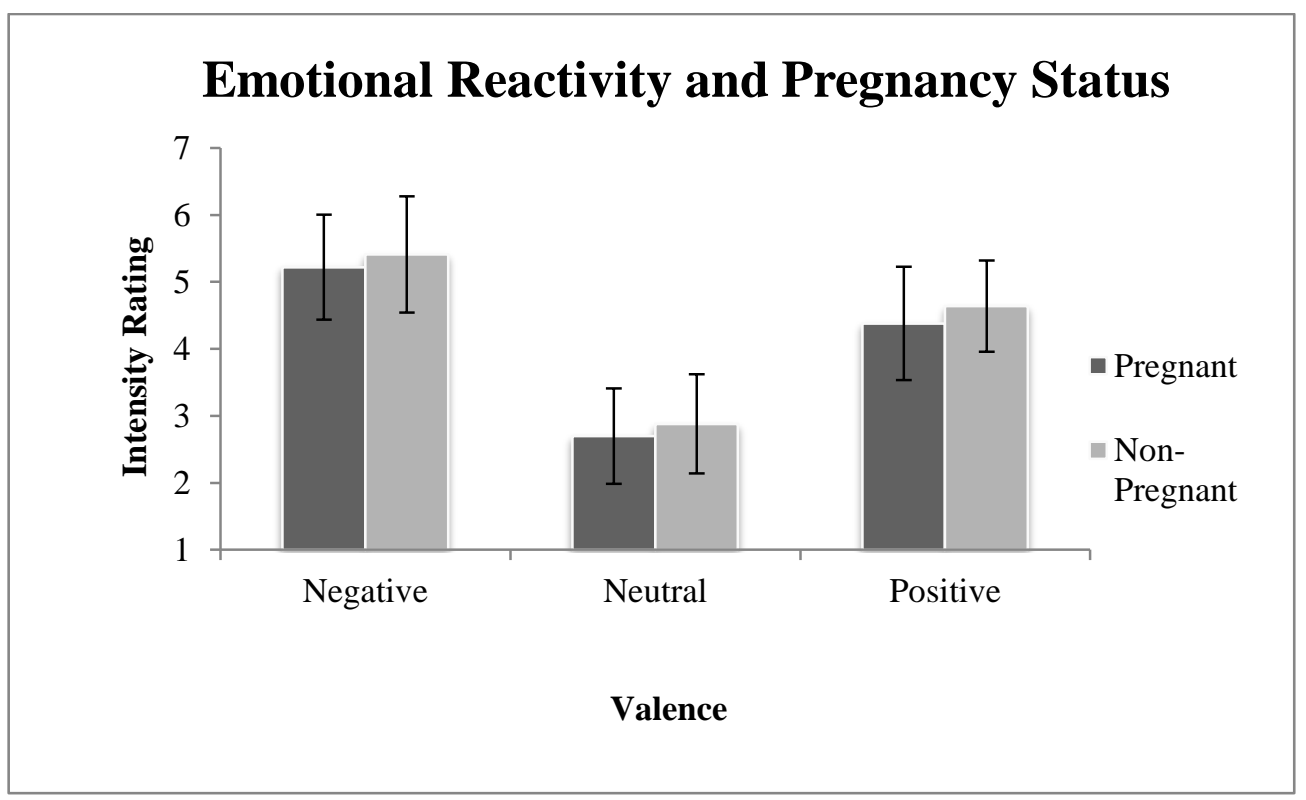


Figure 1. c) Mean intensity ratings for each valence category between previous history of MDD (N=27) and no previous history of MDD (N=50) groups

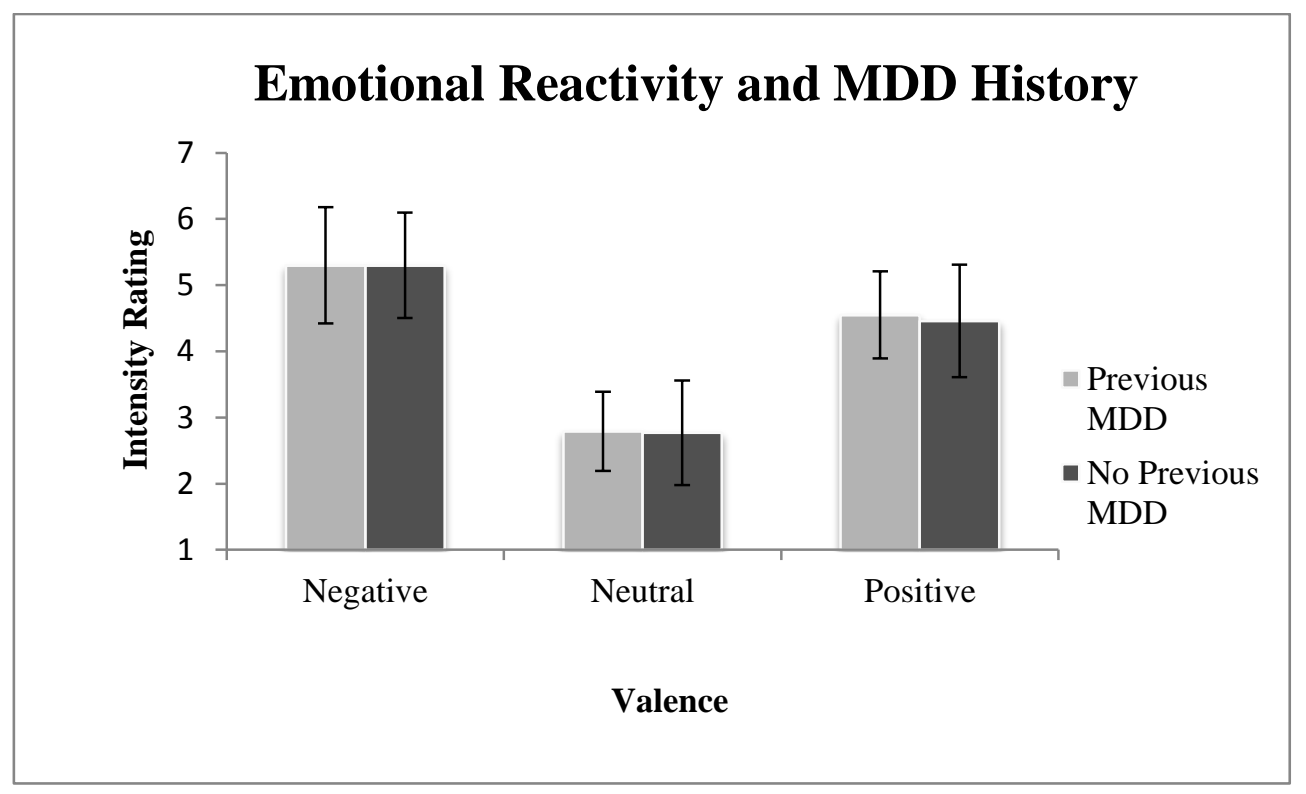


Figure 2. a) Mean normalized memory sensitivity indexes (d') for emotional valence categories across participant groups

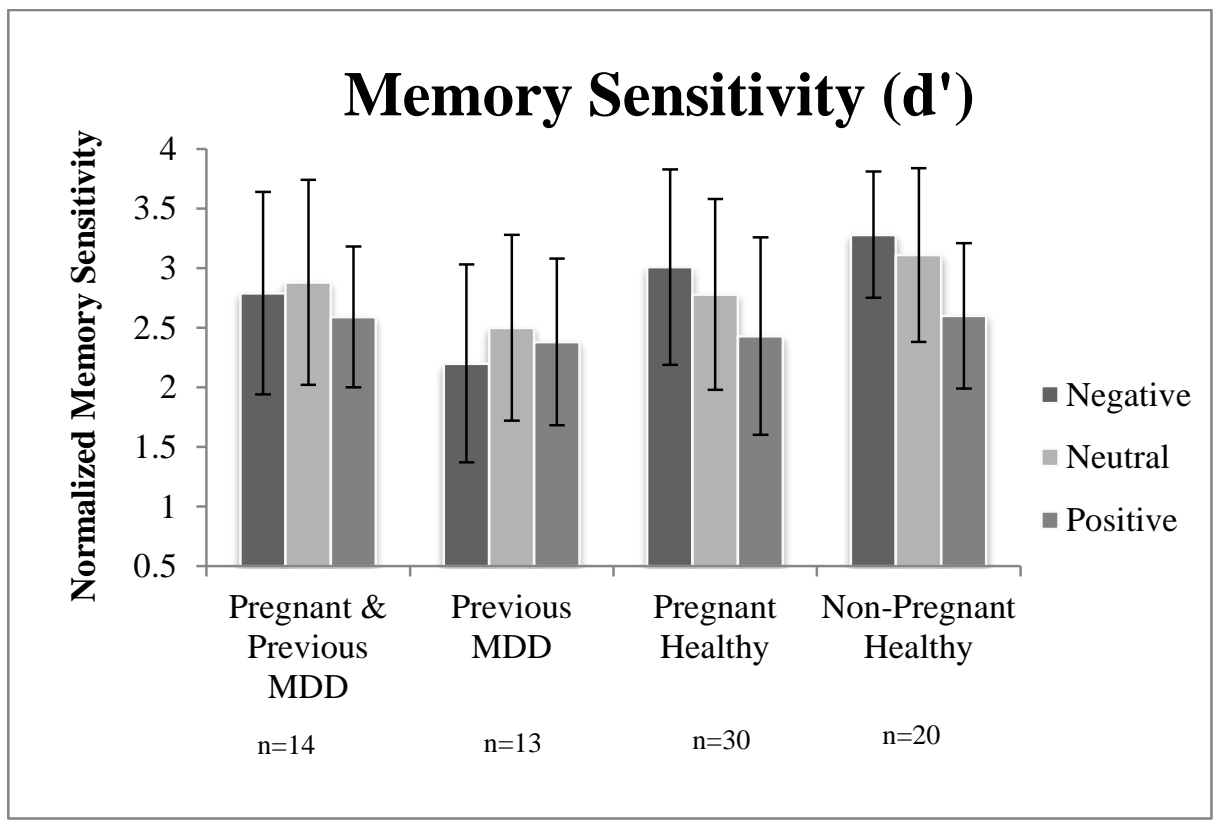


Figure 2. b) Mean normalized memory sensitivity scores for emotional valence categories across pregnant $(\mathrm{N}=44)$ and non-pregnant $(\mathrm{N}=33)$ participant groups

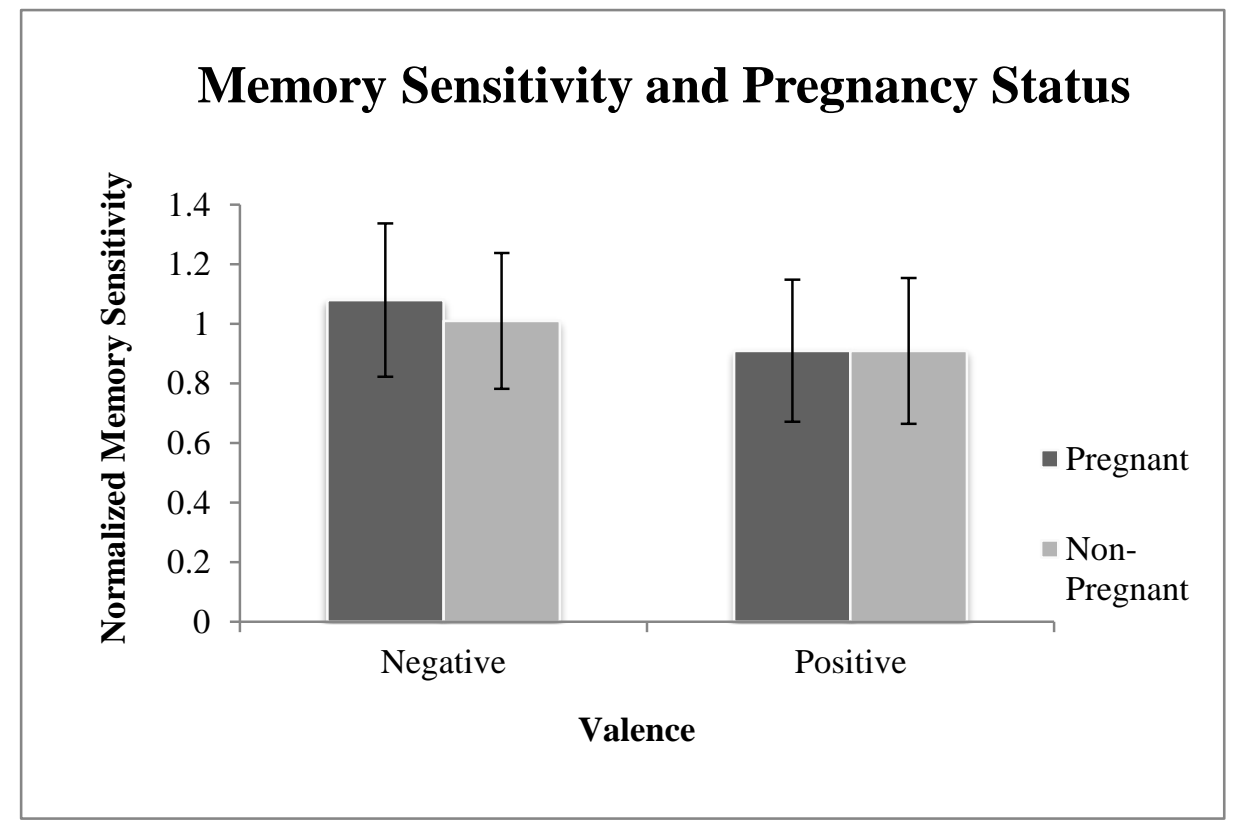


Figure 2. c) Mean normalized memory sensitivity scores for emotional valence categories across participants with $(\mathrm{N}=27)$ and without $(\mathrm{N}=50)$ history of MDD

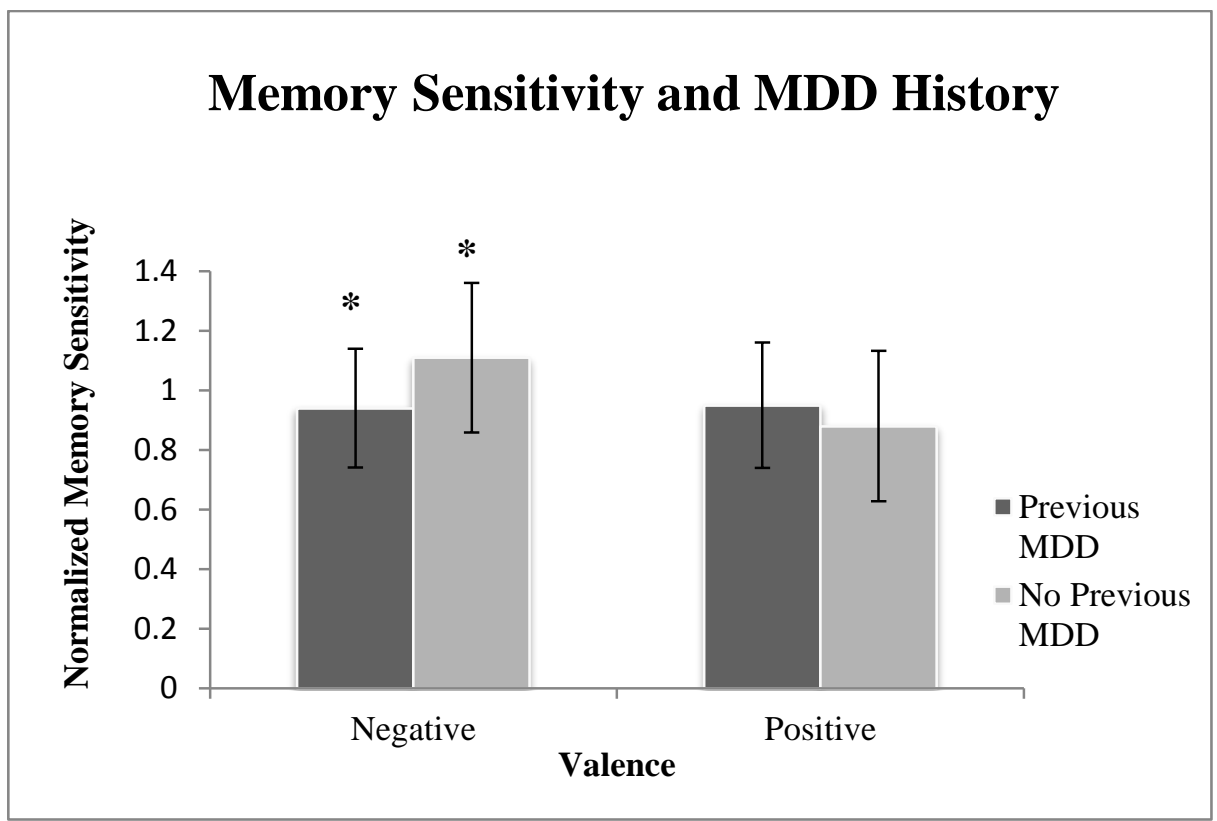

*. The mean difference is significant at the 0.05 level. 
Figure 3. a) Total salivary-free cortisol secretion $\left(\mathrm{AUC}_{\mathrm{G}}\right)$ at baseline (time -1) in pregnant women with history of MDD $(n=14)$

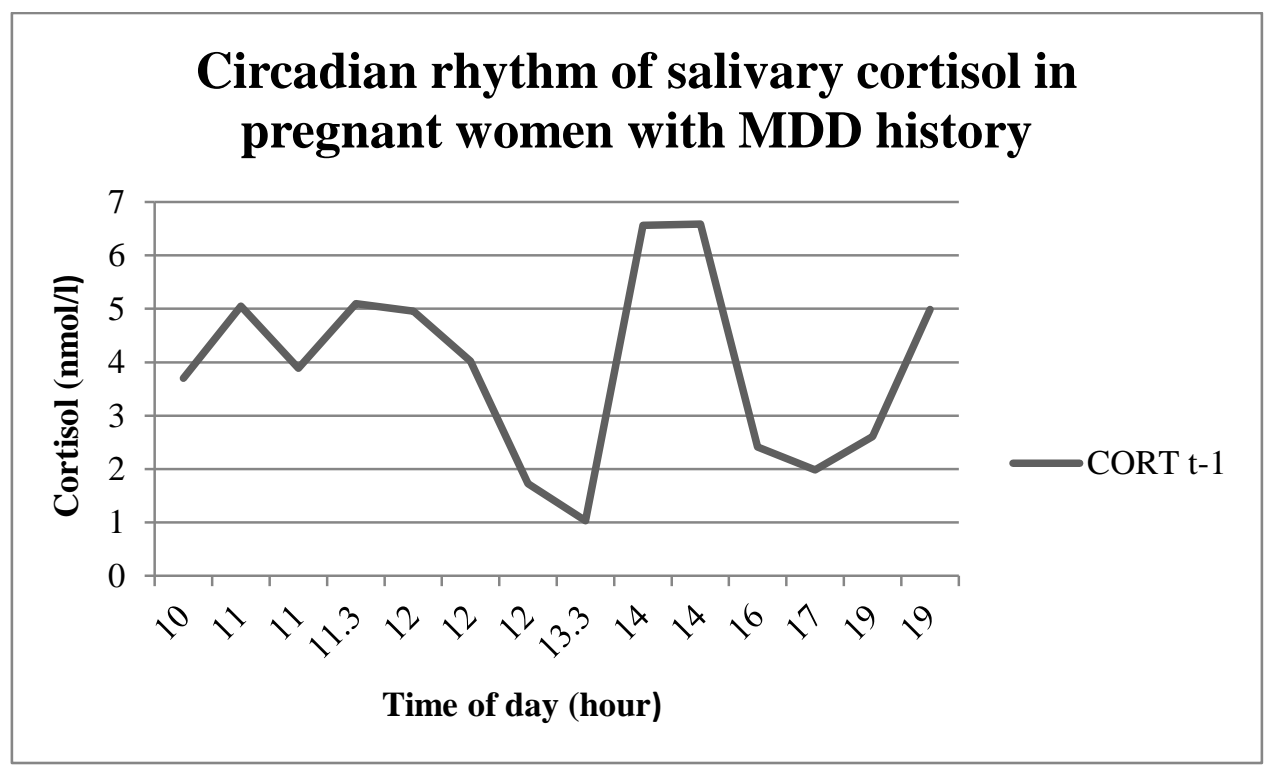


Figure 3. b) Area under the cortisol curve $\left(\mathrm{AUC}_{\mathrm{G}}\right)$ for pregnant women with history of MDD

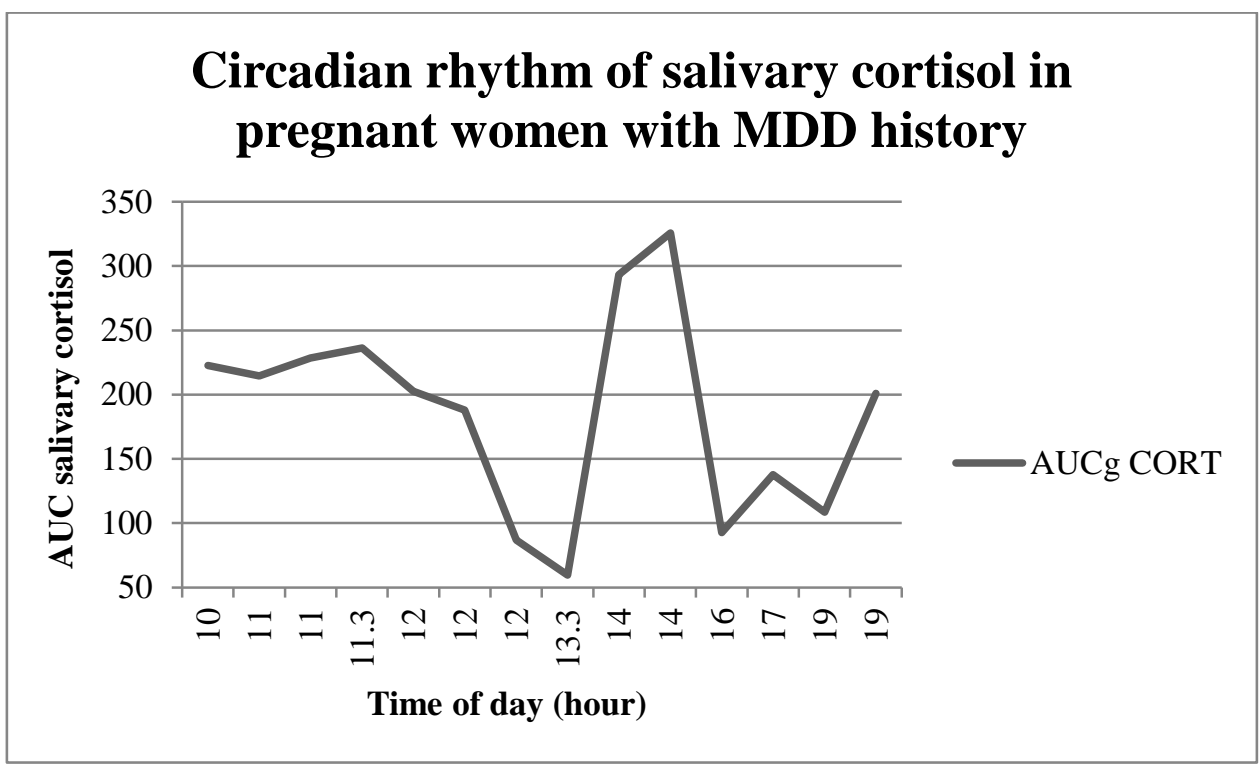


Figure 3. c) Area under the cortisol curve $\left(\mathrm{AUC}_{\mathrm{G}}\right)$ for pregnant women with history of MDD at age of gestation

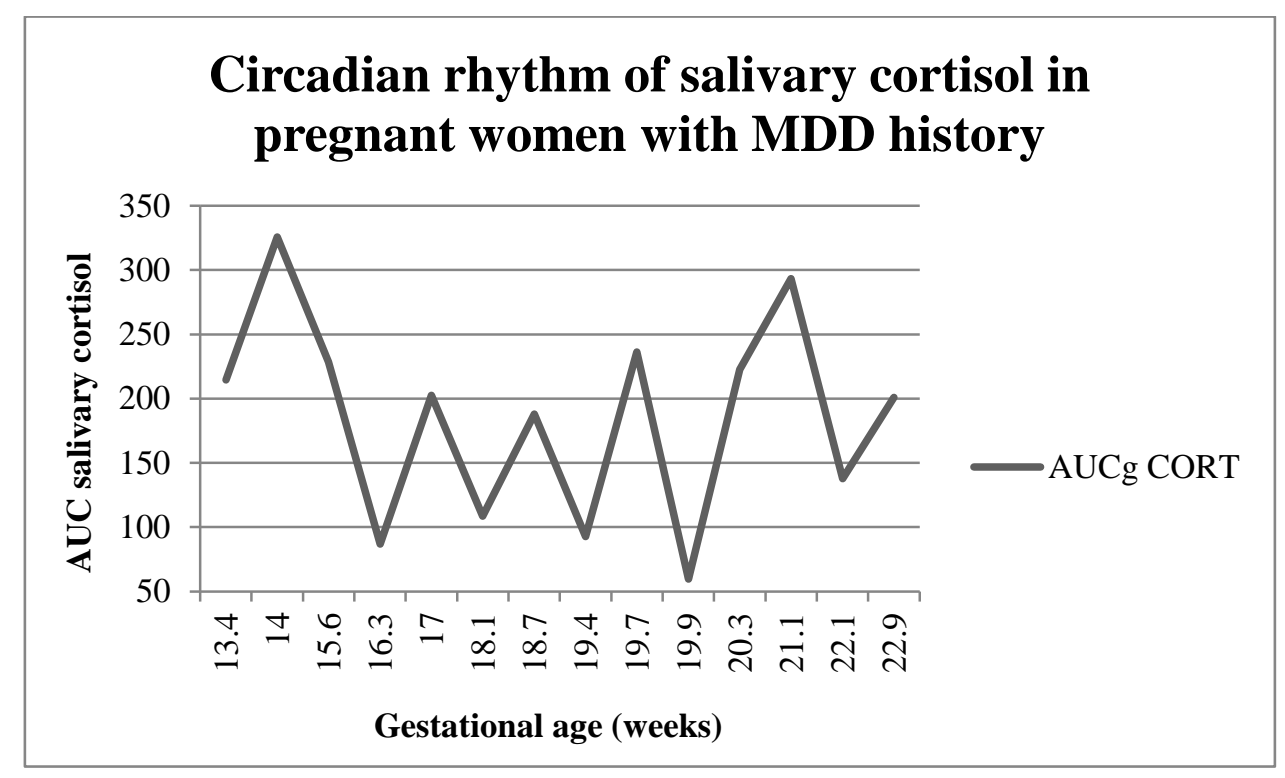


Figure 3. d) Total salivary-free cortisol secretion $\left(\mathrm{AUC}_{\mathrm{G}}\right)$ at baseline (time -1$)$ in nonpregnant women with history of $\operatorname{MDD}(n=12)$

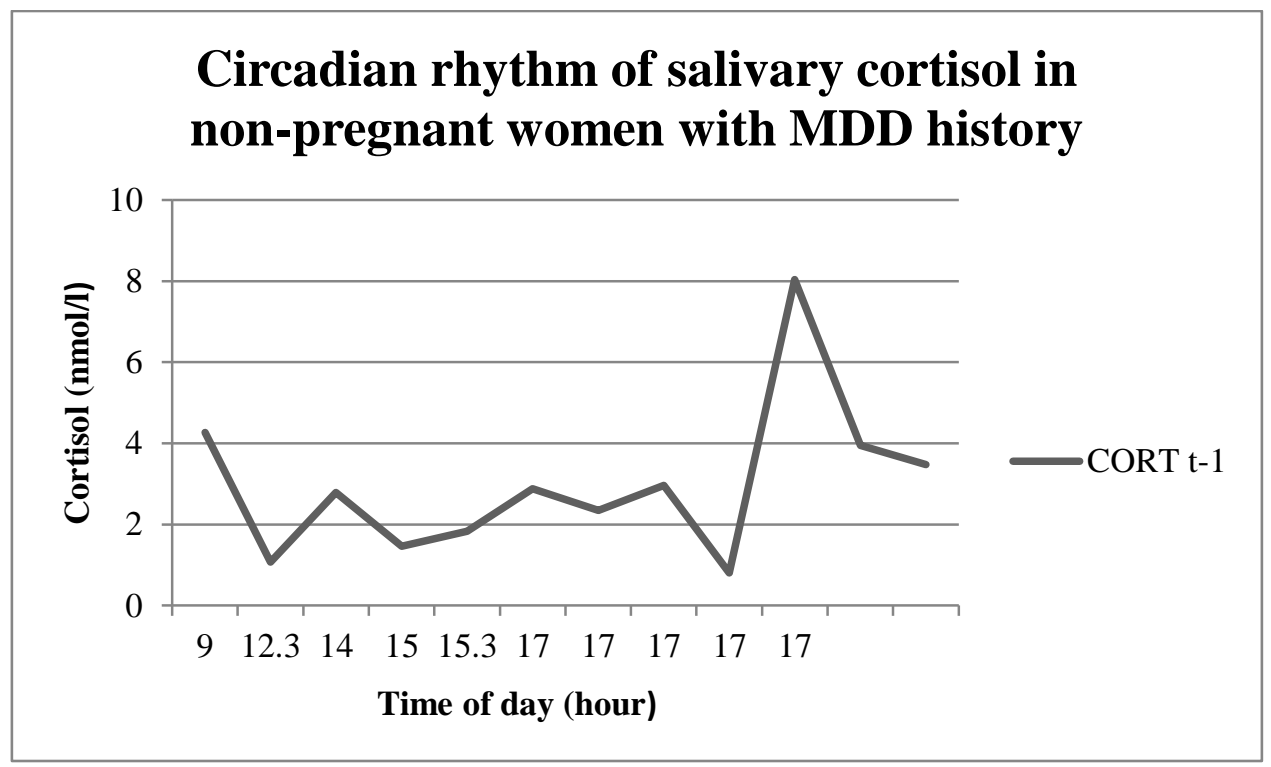


Figure 3. e) Area under the cortisol curve $\left(\mathrm{AUC}_{\mathrm{G}}\right)$ for non-pregnant women with $\mathrm{MDD}$ history

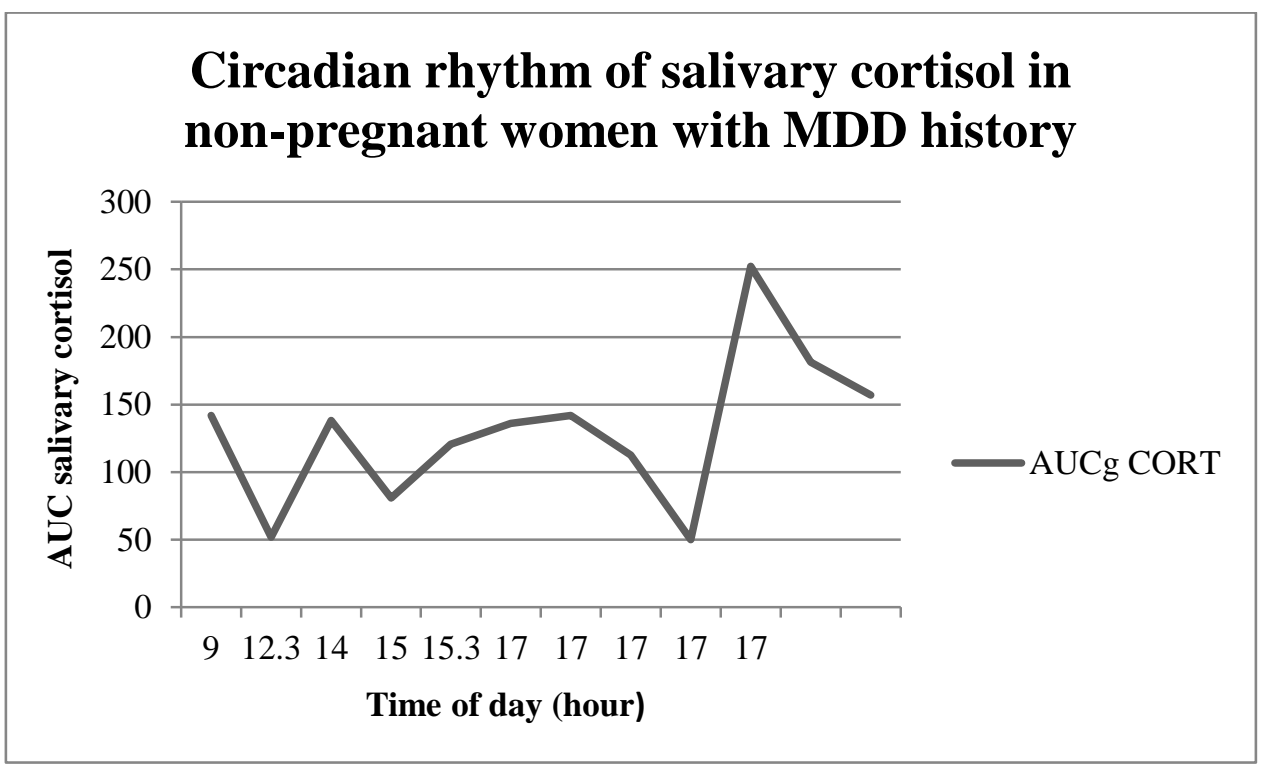


Figure 3. f) Total salivary-free cortisol secretion $\left(\mathrm{AUC}_{\mathrm{G}}\right)$ at baseline (time -1$)$ in pregnant healthy women $(n=28)$

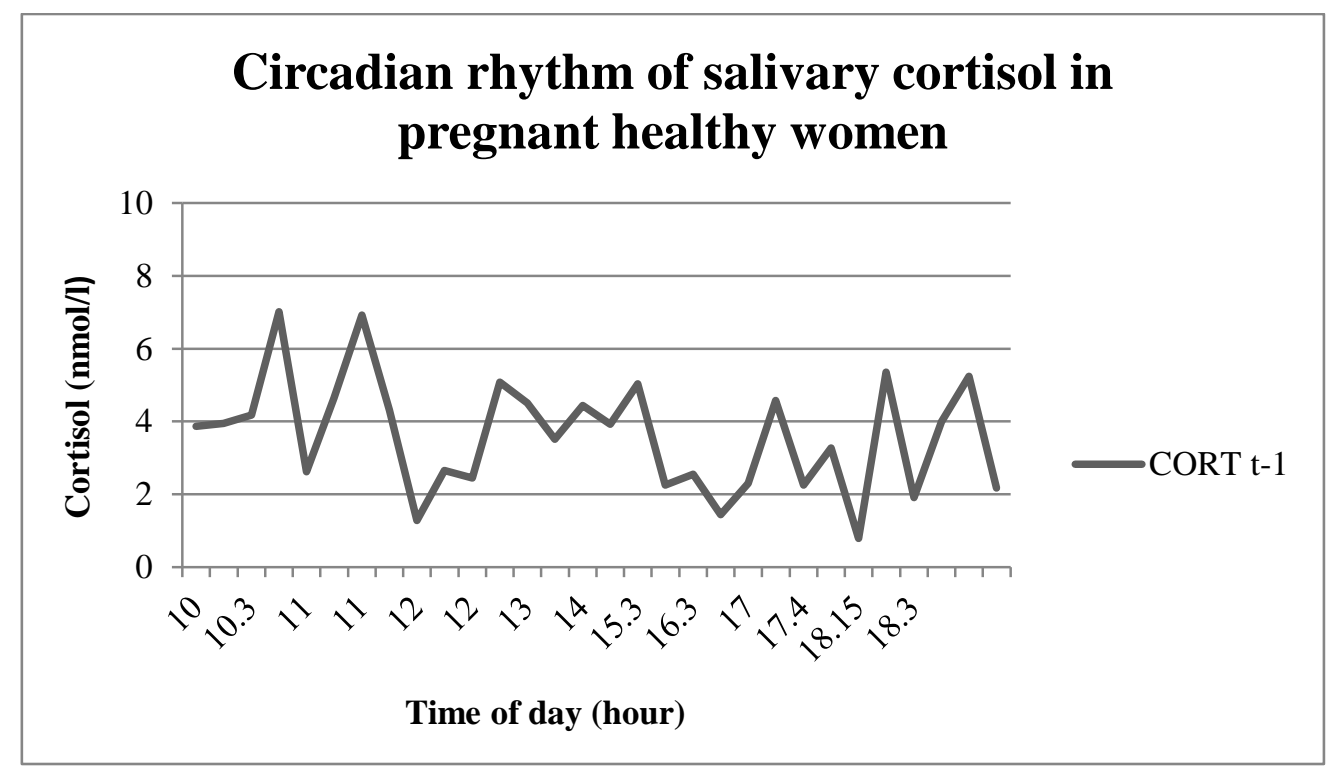


Figure 3. g) Area under the cortisol curve $\left(\mathrm{AUC}_{\mathrm{G}}\right)$ for pregnant healthy women

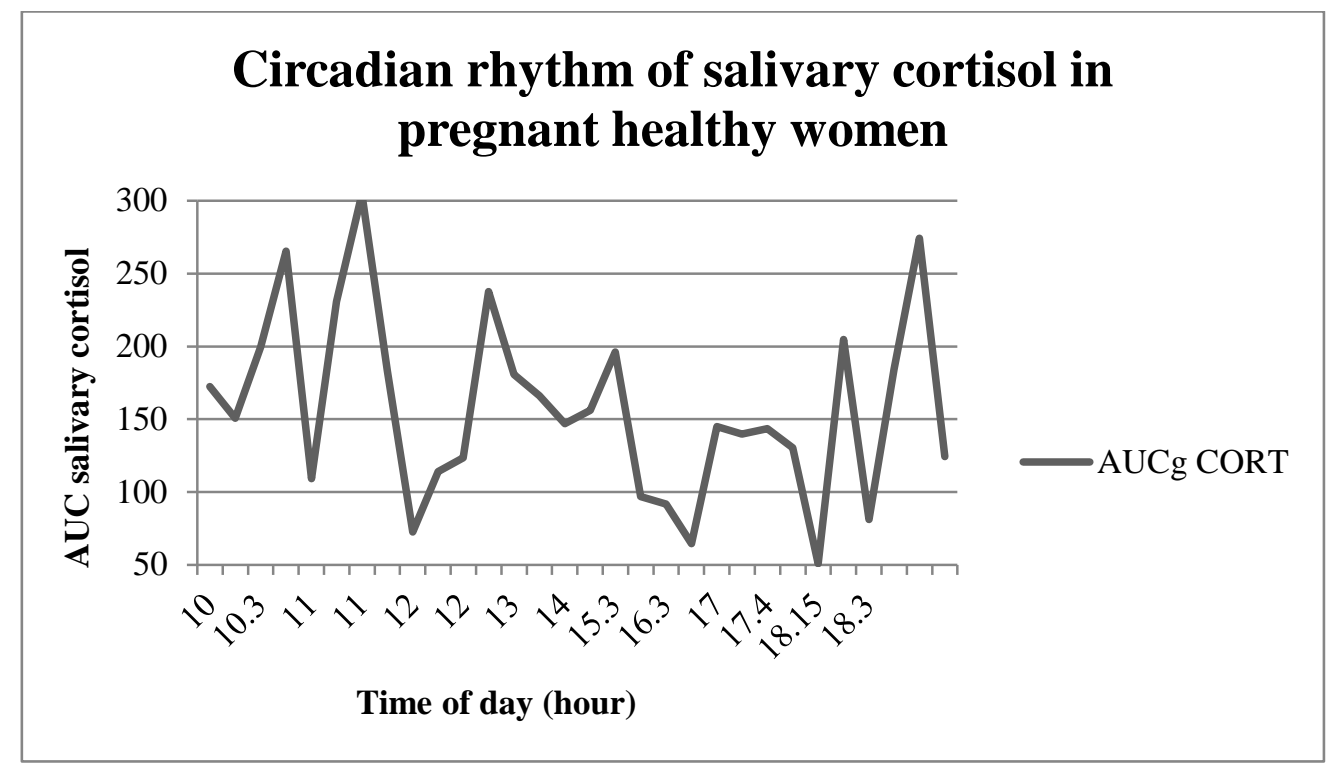


Figure 3. h) Area under the cortisol curve $\left(\mathrm{AUC}_{\mathrm{G}}\right)$ for pregnant healthy women at age of gestation

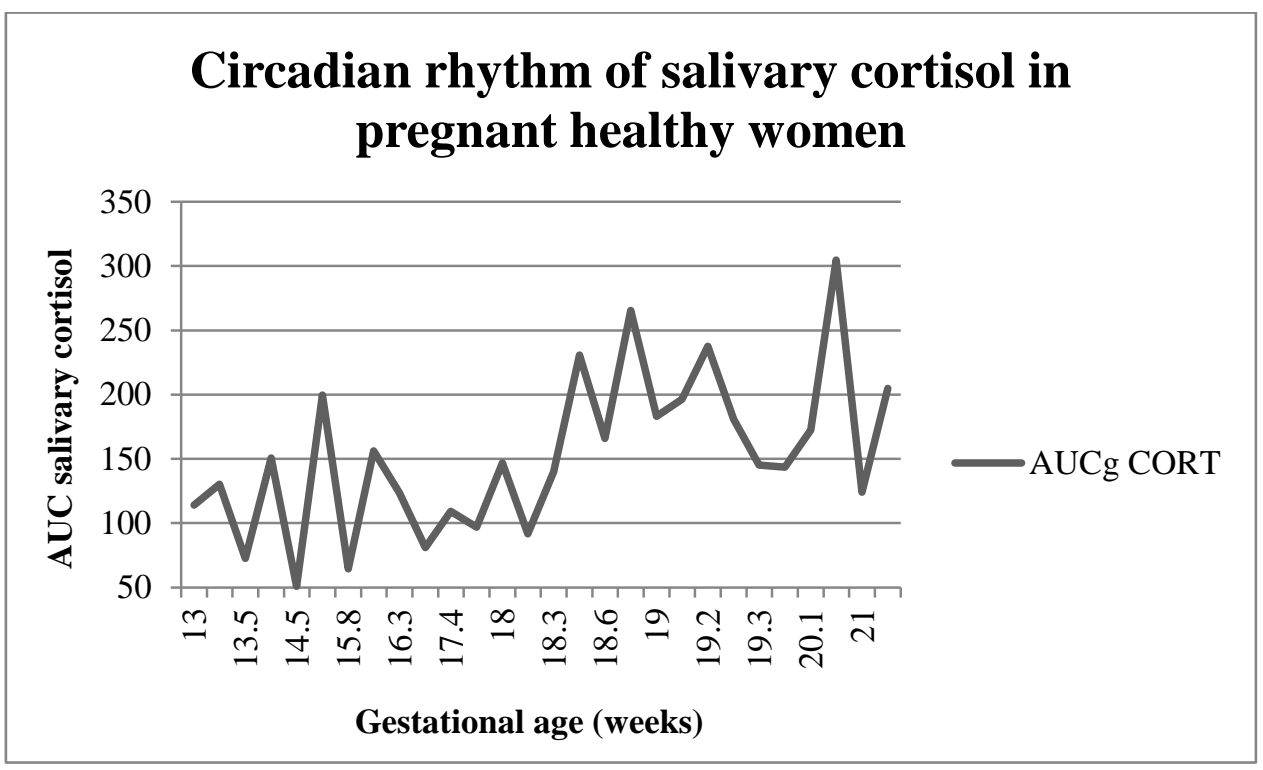


Figure 3. i) Total salivary-free cortisol secretion $\left(\mathrm{AUC}_{\mathrm{G}}\right)$ at baseline (time -1$)$ in nonpregnant healthy women $(n=20)$

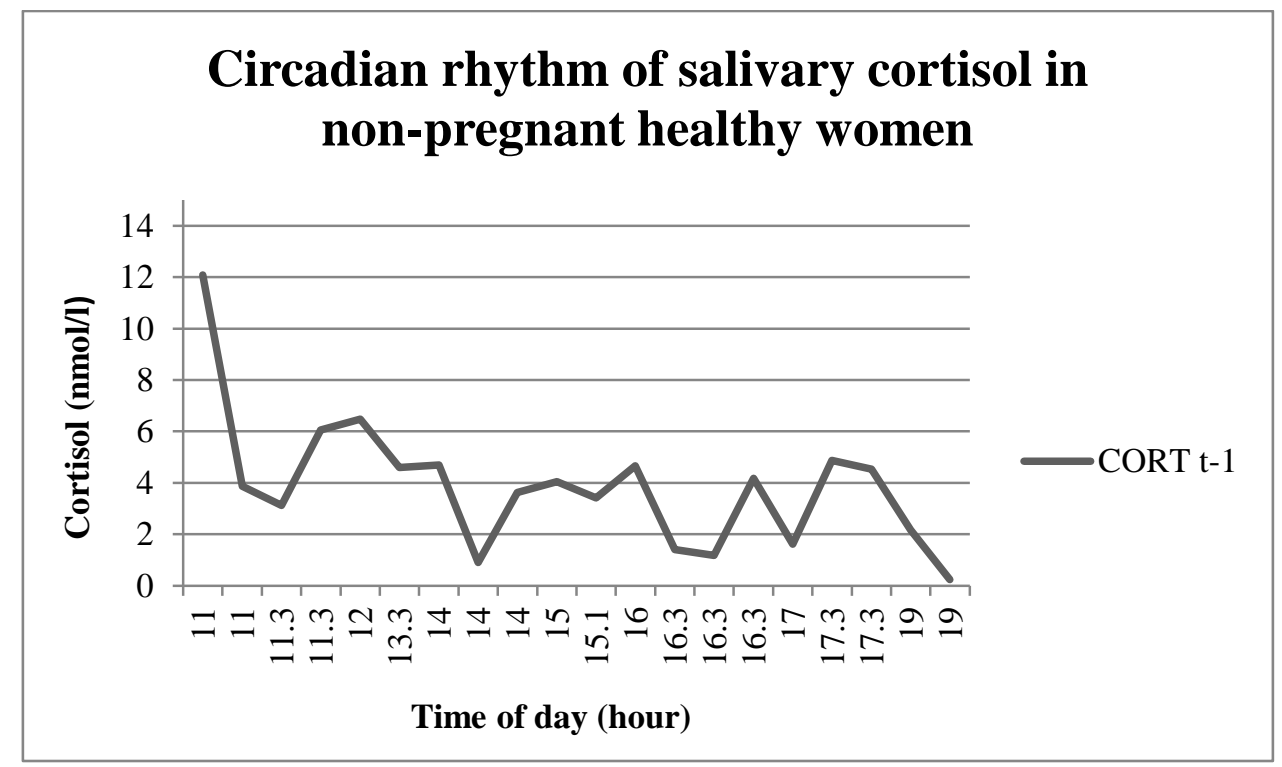


Figure 3. j) Area under the cortisol curve $\left(\mathrm{AUC}_{\mathrm{G}}\right)$ for non-pregnant healthy women

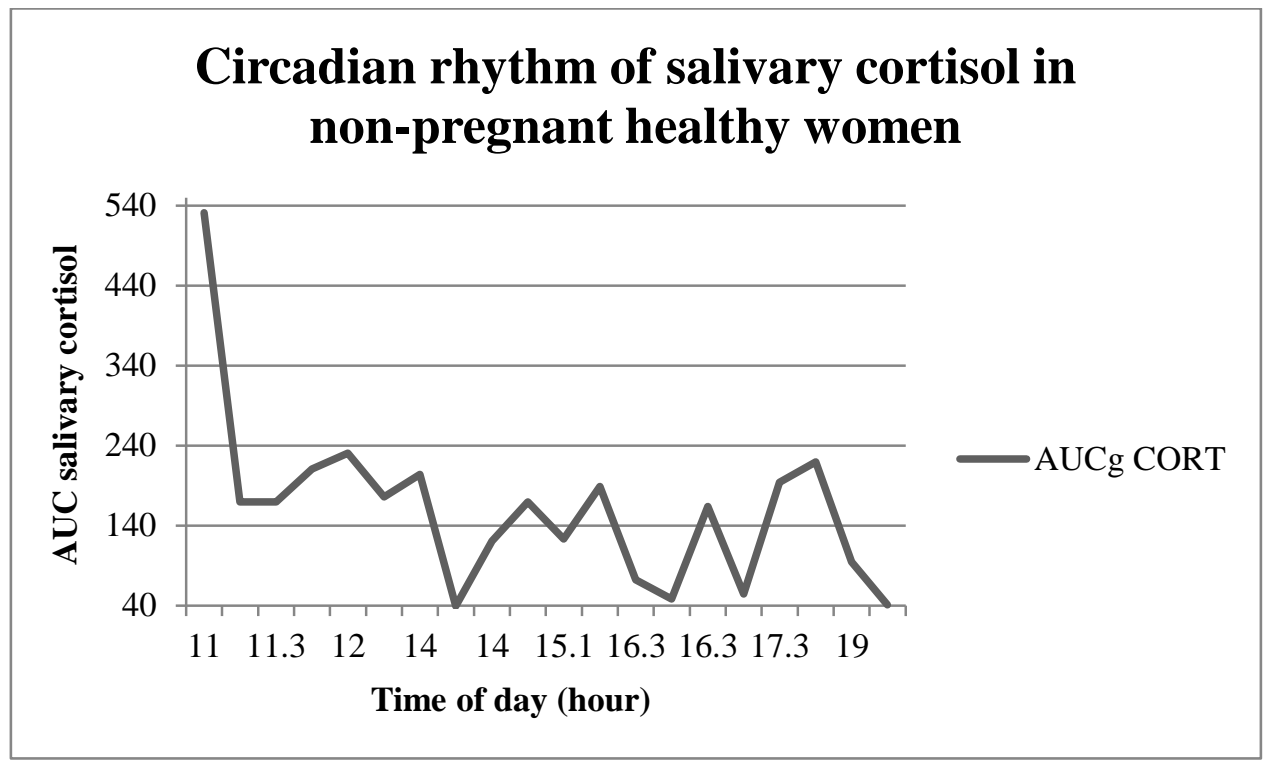




\section{Appendix A: Edinburgh Postnatal Depression Scale (EPDS)}

\section{Edinburgh Postnatal Depression Scale ${ }^{1}$ (EPDS)}

Name:

Your Date of Birth:

Baby's Date of Birth:
Address:

Phone:

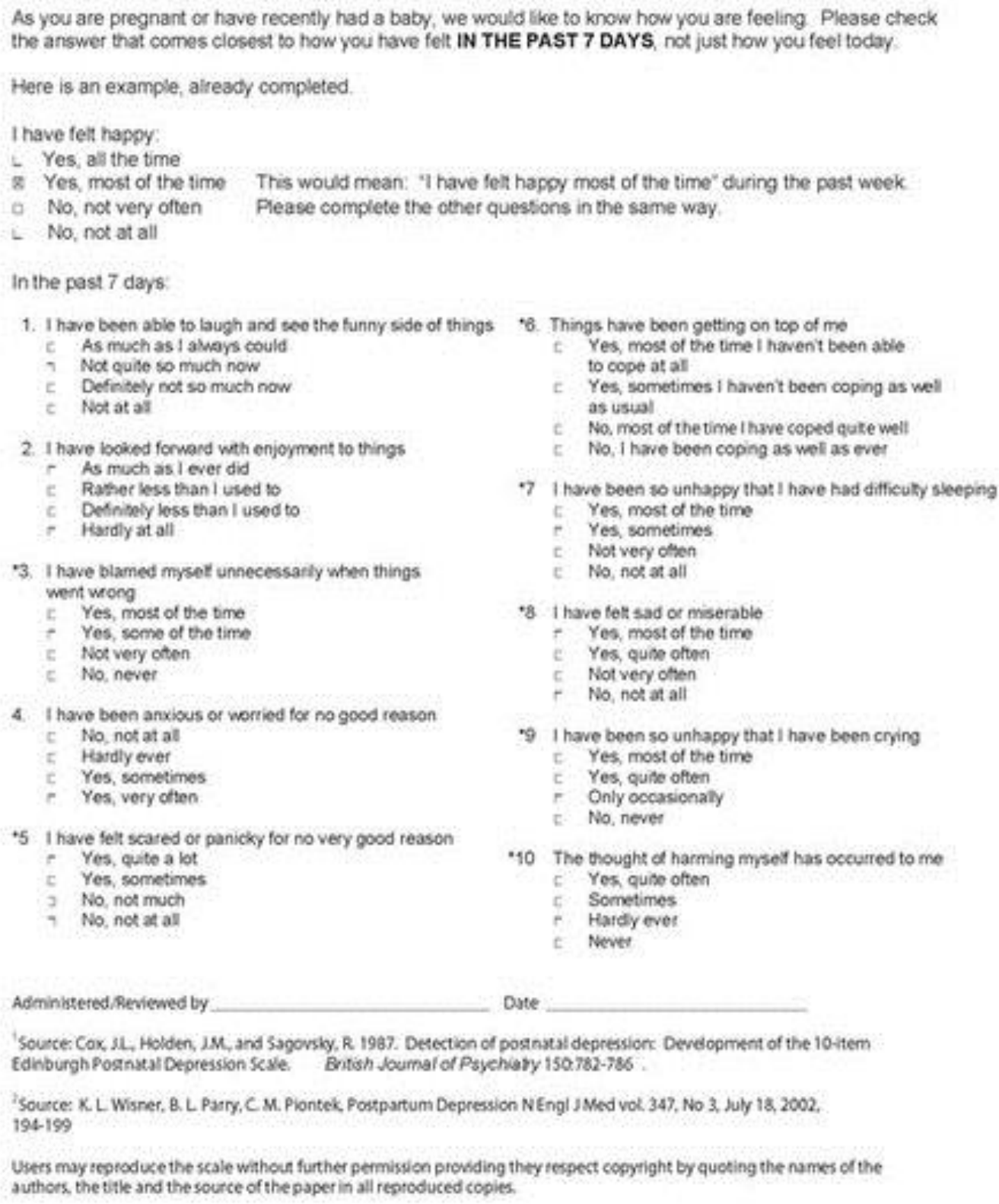

4. Things have been getting on top of me

c. Yes, most of the time I havent been able to cope at all

c. Yes, sometimes i havent been coping as well as usial

c. No most of the time I have coped aute well

c. No, 1 have been coping as well as eve?

77 I have been so unhapgy that I have had difficuly sleeping c. Yes, most of the bime

r. Yes, sometimes

c. Not very ofhen

c. No, not at all

-8 I have felt sad or miserable

- Yes, most of the time

c. Yes, quine olten

c. Not very ohen

r. No, net at all

-9 I have been so unhappy that I have been crying

c. Yes, most of the time

c. Yes, quite often

ir Only occasionaly

c. No, never

-10 The thought of harming myset has oceurred to me

c. Yes quine often

c. Sometimes

if Hardy eve?

c. Neves

Administered/Reviewed by Dute

'Soutce: Cox, JL, Holden, IM, and Sagovsly, R 1987. Desection of postnats depression: Development of the 10-item Edhburgh Pognstai Depression Scsle. Bntsh vouma/ of Psychiary 150.782-785.

'Sourte: K. L. Wisner, 8. L. Parry, C. M. Piontek, Postpartun Depression NEngl J Med vol, 347, No 3, July 18, 2002. 194.199

Usees may :eproduce the scale withou further permissica providing they respest copyright by quoting the names of the authors, the title and the source of the paper in all restoduced copies 


\section{Appendix B:}

\section{Montgomery-Asberg Depression Rating Scale (MADRS)}

Name:

Dato:

\section{Montgomery-Asberg Depression Scale (MADRS)}

Instructlons: The ratings should be based on a cinical intorviaw moving from brosely phrasod cuestions about aymploms to more dotalod ones which allow a procies rating of severity. The rator must docido whother fo rating los on the dolinod scalo stoces $(0,2,4,5)$ of botwoen them $(1,3,5)$. I is mportant to romombor ffrt it is only rate occasions that a deprossed patort is oncountorod who cannot be ralod on the liems in the scale. If datrite answors cannot be olcted from the polonts, all nolovant cluas as wol as infomation from other souroos chould be uesd as a basis for the rating in Ine whe customay clinical proctiog. This scalo may be usod for any time intaval botwoon ratings, be it wookly or otherwise, but this must be rocordod.

\section{Apparent Sadhoss}

Fepresenting dospondony, gloom and despair, (mors than just ordinary transiont low spirts) roflociod in speoch, tacid expression, and posture. Fato on dopth and inablity to brighton up.

No socinoas

Looks dispirlod but does brighton up without dificuly.

Appours sad and unhoppy most of the time.

\section{Extromply degrondort}

Floported Sudhess

Fapresenting reports of dacrosesd mood, rogardless of whather it is rafloctad in appesaranos or not. Includes low spirits, daspondancy or fooling of being bay ond holp without hope. Fale acoording to intansty. duration and fre wesnt to which the mood is reporiad is be influenced by ovants.

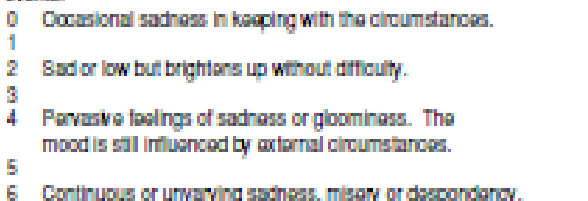

\section{In Inor Tongion}

Fiscresenting foolings of Il-definod discomfort, edginess, inner tumol mounting to ofhor panic, dresd or anguish. Fate acoording to intareity. iroquancy, diration and the oxtont of rassaurance callod tor.

1 Cocasional foolings of edginoas and It dotinod dsoomiort.

4 Continuous fealngs of imar fonsion or intamitiant paric 5 which fo patient can only mastar with some defliculty.

\section{Urrolonting drasd or angulsh. Ovowholming panic}

\section{Paducod Soop}

Fiscresenting the axportance of roduced duration or depth of slose

comparod io he subjocfs own nomal patern whon woll.

of Slosps as usud.

1

2 Sight affeuly dropping of to slosp or slightly roduced Igitit or fftul sloop.

4 slosp rodicod or broken by at losat wo houry.

5

\section{5 los hin wo or three hours slegec}

5. Foduced Appoire

Fepresenting the fooling of loss of appofite oompared whe whan woll. Fisto by loss of desire for food or the nesd is force onesolf to adt.

o Normal or incrased apoetro.

1

$\frac{1}{2}$

3

4 No appottis. Food is tasidoess.

6 Noods porsussion to eat

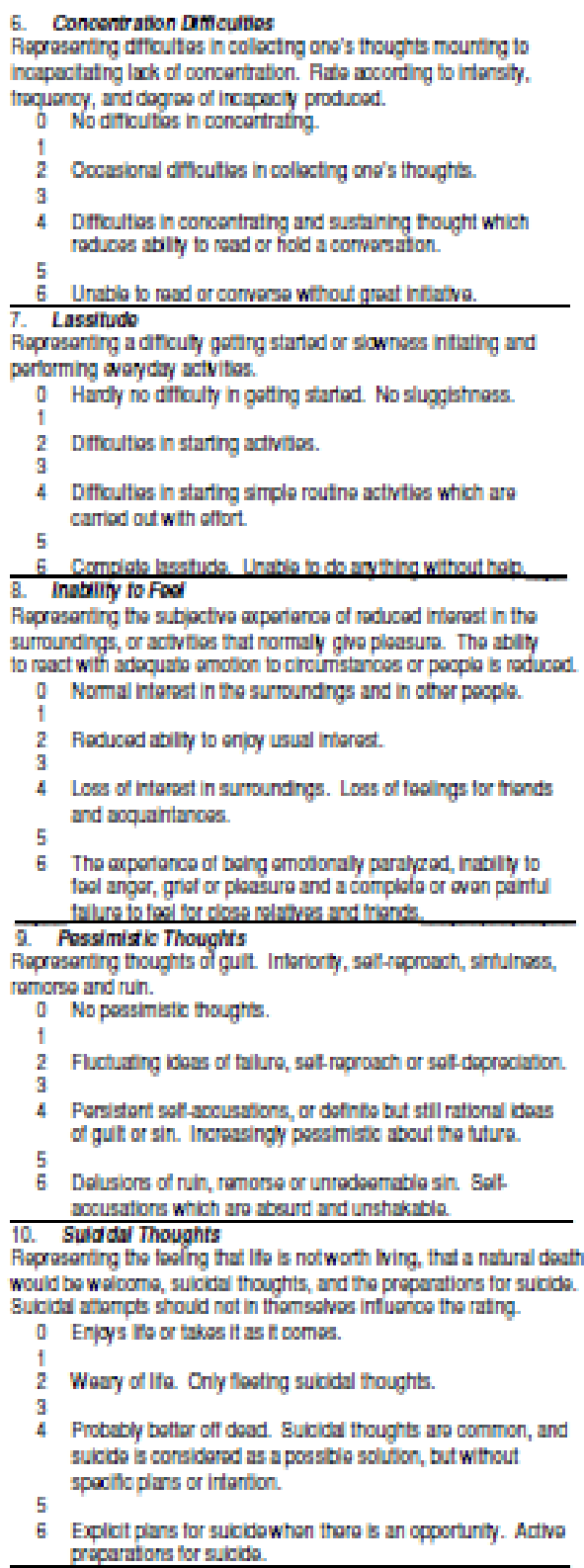

Reprosonting a deflculy goiting startod or slownoss intiating and pertorming avaryday sctvitias.

0 Harcly no diffoulty in gotting startod. No sluggishnoss.

2 Difoultios in starting activitos.

4 Dffoultios in starting simple routne activtios which are carriod out with effort

5

3 inability fo $F$ oo

Reprosenting the subjoctive axportance of rocucod interest in the surroundings, or activisos that normaly give ploasure. The ablity to reast with adocuste anotion to creimstanoes or pooplo is reducod. 0 Nomal intarost in the surroundings and in other posple.

2 Rocucod ability to enjoy usual intorest.

4 Loss of intarost in surroundings. Loas of foolings for frionds and soquaintanose:

6 The axportanoe of being emolonaly paratyzed, inabilty to lool anger, grist or pleasure and a complote or won paint. frlung io foel for closes nolstures and triand:

2. Possimistic Thoughts Raprasanting thoughts of gult. Inforiorty, soll-raprosch, simtulnoss, romorse and run.

(0) No posimiatic thoughts.

1

2 Fluchuaring idass of fallure, sol-raprosch or sol-docrociation.

4 Persigtont sell-accusalons, or definto but still rationd doss of gult or sin. Incroasingly pessimisto about the tuturo.

6 Dolusions of nun, romorse or unrodsomable sin. Solt socusations which aro sbeurd and unshokgtole

10. Suid do Thoughts

Reprosenting the foeing that Ifo is notworh iving, that a nahural doath would be woloome, suicidd thoughts, and the proporations for suicide. sucidar atampts should not in thomselvos infuonos the rating

0 Enjoys ro or takes if as ti comes.

2 Waxy of Ifa. Only lloating suicidal houghts.

4 Probobly bettor off dead. Suicida thoughts aro common, and suicide is considarod as a possble solition, but withoul

5 spocille plars or irtartion.

6 Explicit plans for suicidowhen thare is an opportunity. Activo precaratons for suicide.

Total Scorg: 


\section{Appendix C:}

\section{State-Trait Anxiety Inventory (STAI)}

Subject \#:

Date:

\section{STAI}

Instructions: A number of statements which people have used to describe themselves are given below. Read each statement and then circle the appropriate number to indicate how you feel right now, that is, at this moment. There are no right or wrong answers. Do not spend too much time on any one statement, but give the answer which seems to describe your present feelings best.

\section{Not at all}

1

1. I feel calm.

2. I feel secure.

3. I am tense.

4. I am regretful.

5. I feel at ease.

6. I feel upset.

7. I am presently worrying over possible misfortunes.

8. I feel rested.

9. I feel anxious.

10. I feel comfortable.

11. I feel self-confident.

12. I feel nervous.

13. I am jittery.

14. I feel "high strung".

15. I am relaxed.

16. I feel content.

\section{Somewhat}

2

\section{Moderately so}

3

\section{Very much so \\ 4}

$\begin{array}{llll}1 & 2 & 3 & 4\end{array}$

$\begin{array}{llll}1 & 2 & 3 & 4\end{array}$

$\begin{array}{llll}1 & 2 & 3 & 4\end{array}$

$\begin{array}{llll}1 & 2 & 3 & 4\end{array}$

$\begin{array}{llll}1 & 2 & 3 & 4\end{array}$

$\begin{array}{llll}1 & 2 & 3 & 4\end{array}$

$\begin{array}{llll}1 & 2 & 3 & 4\end{array}$

$\begin{array}{llll}1 & 2 & 3 & 4\end{array}$

$\begin{array}{llll}1 & 2 & 3 & 4\end{array}$

$\begin{array}{llll}1 & 2 & 3 & 4\end{array}$

$\begin{array}{llll}1 & 2 & 3 & 4\end{array}$

$\begin{array}{llll}1 & 2 & 3 & 4\end{array}$

$\begin{array}{llll}1 & 2 & 3 & 4\end{array}$

$\begin{array}{llll}1 & 2 & 3 & 4\end{array}$

$\begin{array}{llll}1 & 2 & 3 & 4\end{array}$

$\begin{array}{llll}1 & 2 & 3 & 4\end{array}$ 
17. I am worried.

$\begin{array}{llll}1 & 2 & 3 & 4\end{array}$

18. I feel over-excited and "rattled".

$\begin{array}{llll}1 & 2 & 3 & 4\end{array}$

19. I feel joyful.

$\begin{array}{llll}1 & 2 & 3 & 4\end{array}$

20. I feel pleasant.

$\begin{array}{llll}1 & 2 & 3 & 4\end{array}$

21. I feel pleasant.

$\begin{array}{llll}1 & 2 & 3 & 4\end{array}$

22. I tire quickly.

$\begin{array}{llll}1 & 2 & 3 & 4\end{array}$

23. I feel like crying.

$\begin{array}{llll}1 & 2 & 3 & 4\end{array}$

24. I wish I could be as happy as others seem to be.

$\begin{array}{llll}1 & 2 & 3 & 4\end{array}$

25. I am losing out on things because I can't make up my mind soon enough.

$\begin{array}{llll}1 & 2 & 3 & 4\end{array}$

26. I feel rested.

$\begin{array}{llll}1 & 2 & 3 & 4\end{array}$

27. I am "calm, cool, and collected".

$\begin{array}{llll}1 & 2 & 3 & 4\end{array}$

28. I feel that difficulties are piling up so that I cannot overcome them.

$\begin{array}{llll}1 & 2 & 3 & 4\end{array}$

29. I worry too much over something that really doesn't matter.

$\begin{array}{llll}1 & 2 & 3 & 4\end{array}$

30. I am happy.

$\begin{array}{llll}1 & 2 & 3 & 4\end{array}$

31. I am inclined to take things hard.

$\begin{array}{llll}1 & 2 & 3 & 4\end{array}$

32. I lack self-confidence.

$\begin{array}{llll}1 & 2 & 3 & 4\end{array}$

33. I feel secure.

$\begin{array}{llll}1 & 2 & 3 & 4\end{array}$

34. I try to avoid facing a crisis or difficulty.

$\begin{array}{llll}1 & 2 & 3 & 4\end{array}$

35. I feel blue.

$\begin{array}{llll}1 & 2 & 3 & 4\end{array}$

36. I am content.

$\begin{array}{llll}1 & 2 & 3 & 4\end{array}$

37. Some unimportant thought runs through my mind and bothers me.

$\begin{array}{llll}1 & 2 & 3 & 4\end{array}$

38. I take disappointments so keenly that I can't put them out of my mind.

$\begin{array}{llll}1 & 2 & 3 & 4\end{array}$

39. I am a steady person.

$\begin{array}{llll}1 & 2 & 3 & 4\end{array}$

40. I get in a state of tension or turmoil as I think over my recent concerns and interests. 


\section{Appendix D:}

\section{Postpartum Depression Predictors Inventory-Revised (PDPI-R)}

TABLE 2

Postpartum Depression Predictors Inventory (PDPI)-Revised and Guide Questions for Its Use

\section{During Pregnancy}

Marital Status

1. Single

2. Married/cohabitating

3. Separated

4. Divorced

5. Widowed

6. Partnered

Socioeconomic status

Low

Middle

High

Self-esteem

Do you feel good about yourself as a person?

Do you feel worthwhile?

Do you feel you have a number of good qualities as a person?

Check One

O

0

○

0

0

○

0

O

O

\section{Prenatal depression}

1. Have you felt depressed during your pregnancy?

If yes, when and how long have you been feeling this way?

If yes, how mild or severe would you consider your depression?

Prenatal anxiety

Have you been feeling anxious during your pregnancy?

If yes, how long have you been feeling this way?

Unplanned/unwanted pregnancy

Was the pregnancy planned?

Is the pregnancy unwanted?

History of previous depression

1. Before this pregnancy, have you ever been depressed?

If yes, when did you experience this depression?

If yes, have you been under a physician's care for this past depression?

If yes, did the physician prescribe any medication for your depression?

1. Do you feel you receive adequate emotional support from your partner?

2. Do you feel you receive adequate instrumental support from your partner (e.g., help with household chores or babysitting)?

3. Do you feel you can rely on your partner when you need help?

4. Do you feel you can confide in your partner?

(repeat same questions for family and again for friends)

Marital satisfaction

1. Are you satisfied with your marriage (or living arrangement)?

2. Are you currently experiencing any marital problems?

3. Are things going well between you and your partner? 


\section{TABLE 2}

\section{Continued}

Life stress

Yes

No

1. Are you currently experiencing any stressful events in your life such as: financial problems marital problems death in the family

serious illness in the family

O

O

moving

$\mathrm{O}$

○

unemployment

O

O

O

job change

After delivery, add the following items Child care stress

1. Is your infant experiencing any health problems?

2. Are you having problems with your baby feeding?

O

O

o

O

3. Are you having problems with your baby sleeping?

Infant temperament

1. Would you consider your baby irritable or fussy?

2. Does your baby cry a lot?

3. Is your baby difficult to console or soothe?

o

O

O

Maternity blues

1. Did you experience a brief period of tearfulness and mood swings during the 1 st week after delivery?
O

O

○

0

O

O

○

\section{COMMENTS:}




\section{Appendix E:}

\section{Pittsburgh Sleep Quality Index (PSQI)}

\section{Appendix. Pittsburgh Sleep Quality Index (PSQI)}

Name___ IO \#___ Date___ Age ___ ___

Instructions:

The following questions relate to your usual sleep habits during the past month only. Your answers should indicate the most accurate reply for the majority of days and nights in the past month. Please answer all questions.

1. During the past month, when have you usually gone to bed at right? USUAL BED TIME

2. During the past month, how long (in minutes) has it usually take you to fall asleep each night? NUMBER OF MINUTES

3. During the past month, when have you usually gotten up in the morning? USUAL GETTING UP TIME

4. During the past month, how many hours of actual sleep did you get at night? (This may be different than the number of hours you spend in bed.)

HOURS OF SLEEP PER NIGHT

For each of the remaining questions, check the one best response. Please answer all questions.

5. During the past month, how often have you had trouble sleeping because you.

(a) Cannot get to sleep within 30 minutes

Not during the Less than Once or Three or more

past month ___ once a week ____ twice a week ____ times a week

(b) Wake up in the middle of the night or earty morning

Not during the Less than Once or Three or more

past month ___ once a week ___ twice a week ___ times a week

(c) Have to get up to use the bathroom

Not during the Less than

past month ____ once a week

Once or

Three or more

(d) Cannot breathe comfortably

Not during the Less than

twice a week

tmes a week.

past month once a week

Once or

twice a wook

Three or more

(e) Cough or snore loudly

Not during the past month

Less than

Once or

times a week

(f) Feel too coid Not during the

once a weei

twice a week

Three or more

past month

Less than

Once or

imes a week

(g) Feel too hot Not during the past month

once a week _

twice a weok

Three or more

Less than

Once or

times a week

(h) Had bad dreams Not during the

once a week

twice a week

Three or more past month

Less than

Once or

times a week

(i) Have pain

Not during the

once a week

wice a week

Three or more

Less than

Once or

bimes a week

past month

once a week

twice a week

Three or more

times a weok 
(i) Other reason(s), please describe

How often during the past month have you had trouble sleeping because of this?

Not during the Less than Once or Three or more

past month

once a week

twice a week

times a week

6. During the past month, how would you rate your sleep quality overall?

Very good

Fairly good

Fairly bad

Very bad

7. During the past month, how often have you taken medicine [prescribed or "over the counter"] to heip you sleep?
Not during the
Less than
Once or
Three of more
past month
once a week ___ wice a week
times a week

8. During the past month, how oten have you had trouble staying awake while driving, eating meals, or engaging in social activity?

Not during the

Less than

Once or

Three or more

past month

once a week __. twice a week times a week

9. During the past month, how much of a problem has it been for you to keep up encugh enthusiasm to get things done?

No problem at all

Only a very slight problem

Somewhat of a problem

A very big problem

10. Do you have a bed partner or roommate?

No bed partner or roommate

Partner/roommate in other room

Parther in same room, but not same bed

Partner in same bed

If you have a roommate or bed partner, ask him/her how oten in the past month you have had..

(a) Loud snoring

Nol during the

past month

Less than

Once or

Three or more

(b) Long pauses between breaths while asieep

Not during the

Less than

past month

once a week

iwice a week

times a week

(c) Legs twitching or jerking while you sleep

Not during the

Less than

past month

once a week

Once or

Three or more

twice a week

times a weok

(c) Episodes of dis Not during the

on or contusion

Once or

Three of more

past month

Less than

times a week

(e) Other restlessness while you sieept please describe

$\begin{array}{llll}\text { Not during the } & \text { Less than } & \text { Once or } & \text { Three or more } \\ \text { past month } & \text { once a week _ } & \text { twice a week _ n times a meek _ }\end{array}$




\section{Appendix F:}

\section{Emotional Task Instructions}

Instructions for emotional reactivity task (ERT):

In this task, you will see a series of 144 images. This task will last for about 45 minutes. You will first be shown a picture for 3 seconds. You will first see a fixation cross before each picture that you are to focus on. Please view the image for the entire time it is on the screen. A screen will appear after each picture asking you to rate its emotional intensity. The scale ranges from 1- not emotional at all, to 7- extremely emotional. You can choose the rating by pressing the number 1 key to move the scale closer to 1 (not emotional at all), and by pressing the number 2 key to move the scale closer to 7 (extremely emotional). Please try to use the entire scale when making your ratings. Stop when the number you choose appears at the bottom of the screen. You will have 7 seconds before the next picture will appear.

Instructions for the emotional memory task (EMT):

In this test you will see a series of 216 images. Some of these images are those you saw last week, others are not. You will first see a fixation cross before each picture that you are to focus on. Please view the image for the entire time it is on the screen. A screen will appear after each picture asking you to indicate if you have seen the picture previously. For each picture, press the number 1 key if you have not seen the picture before. Press the 
number 2 key if you think the picture is familiar but you are not sure you saw it last week. Press the number 3 key if you remember you have seen the picture before.

1. I have not seen this picture before.

2. It looks familiar, but I am not sure.

3. I remember I have seen this picture before. 